\title{
Absolute Stability Analysis of Discrete-Time Systems With Composite Quadratic Lyapunov Functions
}

\author{
Tingshu Hu and Zongli Lin
}

\begin{abstract}
A generalized sector bounded by piecewise linear functions was introduced in a previous paper for the purpose of reducing conservatism in absolute stability analysis of systems with nonlinearity and/or uncertainty. This paper will further enhance absolute stability analysis by using the composite quadratic Lyapunov function whose level set is the convex hull of a family of ellipsoids. The absolute stability analysis will be approached by characterizing absolutely contractively invariant (ACI) level sets of the composite quadratic Lyapunov functions. This objective will be achieved through three steps. The first step transforms the problem of absolute stability analysis into one of stability analysis for an array of saturated linear systems. The second step establishes stability conditions for linear difference inclusions and then for saturated linear systems. The third step assembles all the conditions of stability for an array of saturated linear systems into a condition of absolute stability. Based on the conditions for absolute stability, optimization problems are formulated for the estimation of the stability region. Numerical examples demonstrate that stability analysis results based on composite quadratic Lyapunov functions improve significantly on what can be achieved with quadratic Lyapunov functions.
\end{abstract}

Index Terms-Absolute stability, composite quadratic function, invariant set, piecewise linear sector, saturation.

\section{INTRODUCTION}

A discrete-time system with multiple nonlinear components is described as

$$
x^{+}=A x+B \psi(F x, t)
$$

where $x$ and $x^{+}$stand for $x(t)$ and $x(t+1)$, respectively, $A \in$ $\mathbf{R}^{n \times n}, B \in \mathbf{R}^{n \times m}$, and $F \in \mathbf{R}^{m \times n}$ are constant matrices, and $\psi(\cdot, \cdot): \mathbf{R}^{m} \times \mathbf{Z} \rightarrow \mathbf{R}^{m}$ represents the nonlinearities, possibly time-varying and uncertain. In the classical absolute stability theory, a nonlinear/uncertain/time-varying component is described with a conic sector. This description allows the nonlinear system practically accessible with tools originally developed for linear systems such as frequency analysis, robustness analysis and more recently, the linear matrix ineqaulity (LMI) optimization technique (see, e.g., [1], [4], [7], [22], [24], [26], [27], [32], and [34]). The conic sector takes into account both the nonlinearity and the possible time-varying uncertainty of the

Manuscript received May 4, 2004; revised November 13, 2004 and February 10, 2005. Recommended by Associate Editor M. Kothare. This work supported in part by the National Science Foundation under Grant CMS-0324329.

T. Hu is with the Department of Electrical and Computer Engineering, the University of Massachusetts, Lowell, MA 01854 USA (e-mail: tingshu@gmail.com).

Z. Lin is with the Charles L. Brown Department of Electrical and Computer Engineering, the University of Virginia, Charlottesville, VA 22904-4743 USA (e-mail: zl5y@ virginia.edu).

Digital Object Identifier 10.1109/TAC.2005.849201 component but could be too conservative for a particular component for which more specific properties can be obtained such as an actuator with saturation or dead zone. For this reason, subclasses of the conic sector which impose additional restrictions on the derivative of $\psi(\cdot, \cdot)$ have been considered and less conservative conditions for absolute stability have been derived (see, e.g., [8], [9], [22], [27], and [29].)

Also motivated by the objective of reducing the conservatism of stability analysis, we introduced a generalized sector in [13] for more flexible and more specific description of a nonlinear component. In contrast to using two straight lines to bound a conic sector, we use two odd symmetric piecewise linear functions that are convex or concave over $\mathbf{R}_{\geq 0}$ to bound an (uncertain) nonlinear function. To be specific, we will call the "generalized sector" the piecewise linear sector. Some common nonlinearities, such as saturation (or saturation-like) and dead zone functions can be exactly or arbitrarily closely described with a piecewise linear sector. When global absolute stability is out of the question or is unable to be confirmed, a region of absolute stability has to be estimated. In such a situation, a more detailed description of the nonlinearity by a piecewise linear sector would promise a larger estimate of the stability region than that by a conic sector.

In [13], the region of absolute stability is estimated with absolutely contractively invariant (ACI) ellipsoids and their convex hull for continuous-time systems with one nonlinear component. The objectives of maximizing ACI ellipsoids were formulated into LMI optimization problems. It was also established that if we have a group of ACI ellispoids, then their convex hull is also ACI. Because of this, larger estimates of the stability region can be produced. The main results in [13] were developed using quadratic Lyapunov functions, which have been extensively used for absolute stability and robustness analysis due to numerical issues.

While a piecewise linear sector promises a larger estimate of the stability region than that by the conic sector, the conservatism of absolute stability analysis can be further reduced by exploring nonquadratic Lyapunov functions. Apart from using the Lur'e type Lyapunov functions, an earlier attempt was made in [28] by combining several quadratic functions. Recent years have witnessed an extensive search for nonquadratic Lyapunov functions, among which are piecewise quadratic Lyapunov functions [23], [25], [33], polyhedral Lyapunov functions [2], [5], and homogeneous polynomial Lyapunov functions (HPLFs) [6], [21].

In [16], the composite quadratic Lyapunov function was introduced for enlarging the stability region of saturated linear systems. This type of functions were further explored in [10], 
[20] where the conditions of stability for linear differential inclusions and those for saturated linear systems were significantly improved. The essential difference between [16] and [20] is that, in [16], the invariance of the convex hull of a family of ellipsoids is concluded from the invariance of each individual ellipsoid, while in [20], the invariance of each individual ellipsoid is no longer required. All the results in [10] and [20] were developed for continuous-time systems. Their discrete-time counterparts will be established in this paper as basic tools for absolute stability analysis.

In this paper, we will consider a discrete-time system with multiple nonlinear components, each of which is bounded by a piecewise linear sector. We will use the composite quadratic Lyapunov functions to perform absolute stability analysis for such a system. The first step toward this goal (contained in Section III) is to transform the absolute stability analysis problem into the stability analysis of an array of saturated linear systems. This is achieved by describing a piecewise linear function with an array of saturation functions. The second step (in Section IV) is to derive stability conditions for a saturated linear system by using composite quadratic functions. This is achieved by first developing a stability condition for linear difference inclusions (LDIs) and then obtaining a regional LDI description for the saturated linear system. The last step (in Section V) is to put together the conditions of stability for all the saturated linear systems into the condition of absolute stability for the system with a piecewise linear sector condition. In Section V, we also formulate optimization problems for enlarging the estimate of the stability region and use two examples to demonstrate the advantage of using composite quadratic functions over using quadratic functions. In particular, the estimate of the stability region for each case is significantly enlarged by using composite quadratic functions.

The problems in this paper are formulated under the discretetime setting. However, all the results can be readily extended to continuous-time systems.

Notation:

- For two integers $k_{1}, k_{2}, k_{1}<k_{2}$, we denote $I\left[k_{1}, k_{2}\right]=\left\{k_{1}, k_{1}+1, \ldots, k_{2}\right\}$.

- We use sat $(\cdot)$ to denote the standard saturation function, i.e., for $u \in \mathbf{R}^{m},[\operatorname{sat}(u)]_{i}=$ $\operatorname{sgn}\left(u_{i}\right) \min \left\{1,\left|u_{i}\right|\right\}$.

- For $N$ vectors $x^{i} \in \mathbf{R}^{n}, i \in I[1, N]$, we use $\operatorname{co}\left\{x^{i}\right.$ : $i \in I[1, N]\}$ to denote the convex hull of these vectors, i.e.,

$\operatorname{co}\left\{x^{i}: i \in I[1, N]\right\}:=\left\{\sum_{i=1}^{N} \gamma_{i} x^{i}: \sum_{i=1}^{N} \gamma_{i}=1, \gamma_{i} \geq 0\right\}$.

- For $N$ functions $\psi^{i}: \mathbf{R}^{n} \rightarrow \mathbf{R}^{m}, i \in I[1, N]$, we use $\operatorname{co}\left\{\psi^{i}: i \in I[1, N]\right\}$ to denote the set of functions $\psi: \mathbf{R}^{n} \rightarrow \mathbf{R}^{m}$ such that

$$
\psi(x) \in \operatorname{co}\left\{\psi^{i}(x): i \in I[1, N]\right\} \quad \forall x \in \mathbf{R}^{n} .
$$

- For a matrix $F \in \mathbf{R}^{m \times n}$, denote

$$
\mathcal{L}(F):=\left\{x \in \mathbf{R}^{n}:|F x|_{\infty} \leq 1\right\}
$$

- Let $P=P^{T} \in \mathbf{R}^{n \times n}$ be a positive-definite matrix. For a positive number $\rho$, denote

$$
\mathcal{E}(P, \rho):=\left\{x \in \mathbf{R}^{n}: x^{T} P x \leq \rho\right\} .
$$

For simplicity, we use $\mathcal{E}(P)$ to denote $\mathcal{E}(P, 1)$.

\section{Generalized Sector And Absolute Stability}

\section{A. Concave and Convex Functions}

The generalized sector, as introduced in [13], is defined in terms of two concave/convex functions. Given a scalar function $\phi: \mathbf{R} \rightarrow \mathbf{R}$. Assume that

1) $\phi(\cdot)$ is continuous, piecewise differentiable and $\phi(0)=0$

2) $\quad \phi(\cdot)$ is odd symmetric, i.e., $\phi(-u)=-\phi(u)$.

A function $\phi(u)$ satisfying the previous assumption is said to be concave if it is concave for $u \geq 0$ and is said to be convex if it is convex for $u \geq 0$. These definitions are made for simplicity. It should be understood by odd symmetry that a concave function is convex for $u \leq 0$ and a convex function is concave for $u \leq 0$.

\section{B. The Generalized Sector and Absolute Stability: Definitions}

Consider the system

$$
x^{+}=A x+B \psi(F x, t)
$$

where $A \in \mathbf{R}^{n \times n}, B \in \mathbf{R}^{n \times m}, F \in \mathbf{R}^{m \times n}$ are given matrices, and $\psi(\cdot, \cdot): \mathbf{R}^{m} \times \mathbf{Z} \rightarrow \mathbf{R}^{m}$ is a decoupled vector function, i.e.,

$$
\psi(u, t)=\left[\begin{array}{llll}
\phi_{1}\left(u_{1}, t\right) & \phi_{2}\left(u_{2}, t\right) \quad \ldots & \phi_{m}\left(u_{m}, t\right)
\end{array}\right]^{T}
$$

and $\phi_{i}(\cdot, \cdot): \mathbf{R} \times \mathbf{Z} \rightarrow \mathbf{R}$. Our objective is to estimate the stability region of (2) with invariant level sets of a certain Lyapunov function.

Let $V: \mathbf{R}^{n} \rightarrow \mathbf{R}$ be a positive-definite Lyapunov function candidate. Given a positive number $\rho$, a level set of $V$ is

$$
L_{V}(\rho):=\left\{x \in \mathbf{R}^{n}: V(x) \leq \rho\right\}
$$

The level set $L_{V}(\rho)$ is said to be contractively invariant for (2) if

$$
\Delta V(x, t)=V(A x+B \psi(F x, t))-V(x)<0
$$

for all $x \in L_{V}(\rho) \backslash\{0\}$ and $t \in \mathbf{Z}$. Clearly, if $L_{V}(\rho)$ is contractively invariant, then it is inside the stability region. If $V(x)$ is a quadratic function $x^{T} P x$, then $L_{V}(\rho)=\mathcal{E}(P, \rho)$.

In the aforementioned definition of contractive invariance, the nonlinear function $\psi(u, t)$ is assumed to be known. For practical reasons, we would like to study the invariance of a level set for a class of nonlinear functions, for example, a class of $\psi(u, t)$, every component of which is bounded by a pair of convex/concave nonlinear functions, i.e.,

$$
\begin{aligned}
\phi_{i}\left(u_{i}, t\right) & \in \operatorname{co}\left\{\breve{\phi}_{i}\left(u_{i}\right), \bar{\phi}_{i}\left(u_{i}\right)\right\} \quad \forall u_{i} \in \mathbf{R} \\
t & \in \mathbf{Z}, \quad i \in I[1, m]
\end{aligned}
$$


where $\breve{\phi}_{i}(\cdot)$ and $\bar{\phi}_{i}(\cdot)$ are known scalar functions. We use $\operatorname{co}\left\{\breve{\phi}_{i}, \bar{\phi}_{i}\right\}$ to denote the generalized sector for the $i$ th component of $\psi(\cdot, \cdot)$. The multivariable sector for $\psi(\cdot, \cdot)$ is the convex hull of $2^{m}$ decoupled functions

$$
\begin{aligned}
\psi^{j}(u) & =\left[\begin{array}{llll}
\phi_{1}^{j}\left(u_{1}\right) & \phi_{2}^{j}\left(u_{2}\right) & \ldots & \phi_{m}^{j}\left(u_{m}\right)
\end{array}\right]^{T} \\
j & \in I\left[1,2^{m}\right]
\end{aligned}
$$

where $\phi_{i}^{j}=\bar{\phi}_{i}$ or $\breve{\phi}_{i}$. We denote this multivariable sector as $\operatorname{co}\left\{\psi^{j}: j \in I\left[1,2^{m}\right]\right\}$. In general case, we may also consider a sector as the convex hull of $N$ decoupled functions $\psi^{j}, \operatorname{co}\left\{\psi^{j}\right.$ : $j \in I[1, N]\}$. We say that $\psi(\cdot, \cdot)$ satisfies a generalized sector condition if

$$
\psi(u, t) \in \operatorname{co}\left\{\psi^{j}(u): j \in I[1, N]\right\} \quad \forall u \in \mathbf{R}^{m}, \quad t \in \mathbf{Z}
$$

and denote it as $\psi \in \operatorname{co}\left\{\psi^{j}: j \in I[1, N]\right\}$.

Definition 1: A level set $L_{V}(\rho)$ is said to be absolutely contractively invariant (ACI) over the sector $\operatorname{co}\left\{\psi^{j}: j \in I[1, N]\right\}$ if it is contractively invariant for (2) under every possible $\psi(\cdot, \cdot)$ satisfying the generalized sector condition.

Clearly, if $L_{V}(\rho)$ is ACI, then every trajectory starting from it will converge to the origin under any $\psi(\cdot, \cdot)$ satisfying the generalized sector condition. Hence, $L_{V}(\rho)$ is a region of absolute stability. We may use more general functions to define the boundary of a sector to capture more details about the nonlinear components. As was explained in [13], the reason that we have chosen concave/convex functions is for the simplicity and completeness of the results they lead to. If we use other more general functions as the boundaries, the condition for ACI may be hard to describe or numerically nontractable. We may also choose asymmetric functions or even symmetric functions. We have settled on odd symmetric functions since we will be focusing on level sets which are symmetric about the origin. Let us next state a simple result.

Lemma 1: Assume that the Lyapunov function $V(x)$ is convex. Given a level set $L_{V}\left(\rho_{0}\right)$ and a sector $\operatorname{co}\left\{\psi^{j}: j \in\right.$ $I[1, N]\} . L_{V}\left(\rho_{0}\right)$ is ACI over the sector if and only if it is contractively invariant for

$$
x^{+}=A x+B \psi^{j}(F x)
$$

for every $j \in I[1, N]$.

Proof: The "only if" part is obvious. Now, we assume that $L_{V}\left(\rho_{0}\right)$ is contractively invariant for (5) for every $j \in I[1, N]$. Consider any $\psi \in \operatorname{co}\left\{\psi^{j}: j \in I[1, N]\right\}$. We need to show that $L_{V}\left(\rho_{0}\right)$ is contractively invariant for

$$
x^{+}=A x+B \psi(F x, t) .
$$

Let $x$ be such that $V(x)=\rho \in\left(0, \rho_{0}\right]$. Since $L_{V}\left(\rho_{0}\right)$ is contractively invariant for (5), we have

$$
V\left(A x+B \psi^{j}(F x)\right)<V(x)=\rho \quad \forall j \in I[1, N] .
$$

Since $V$ is a convex function and $\psi(F x, t) \in \operatorname{co}\left\{\psi^{j}(F x): j \in\right.$ $I[1, N]\}$, we have

$$
\begin{aligned}
& V(A x+B \psi(F x, t)) \\
& \leq \max \left\{V\left(A x+B \psi^{j}(F x)\right): j \in I[1, N]\right\}<V(x)
\end{aligned}
$$

which implies the contractive invariance of $L_{V}\left(\rho_{0}\right)$ for (6).
A similar result exists for continuous-time systems, where the differentiability of $V$ is required instead of the convexity in Lemma 1.

We will restrict our attention to the level sets of convex Lyapunov functions. By Lemma 1, the absolute contractive invariance of a level set is equivalent to its contractive invariance under every vertex function $\psi^{j}$. Since a nonlinear function can be well approximated with a piecewise linear function, we will focus our attention on the case where the components of $\psi^{j}$ are piecewise linear convex/concave functions. This will make the stability analysis problems numerically approachable. In this case, we call the generalized sector a piecewise linear sector.

\section{A Class of Piecewise Linear Functions}

Consider the class of piecewise linear functions

$$
\phi(u)= \begin{cases}k_{0} u, & \text { if } u \in\left[0, b_{1}\right] \\ k_{1} u+c_{1}, & \text { if } u \in\left(b_{1}, b_{2}\right] \\ \vdots & \\ k_{N} u+c_{N}, & \text { if } u \in\left(b_{N}, \infty\right)\end{cases}
$$

where $0<b_{1}<b_{2}<\cdots<b_{N}$. The values of $\phi(u)$ for $u<0$ can be determined by odd symmetry. It is easy to see that if $\phi(u)$ is concave, then $k_{0}>k_{1}>k_{2}>\cdots>k_{N}>-\infty$ and $0<c_{1}<c_{2}<\cdots<c_{N}$. In the case that $k_{0}>0$ and $k_{N}=0$, $\phi(u)$ is a saturation like function with a saturation bound $c_{N}$. If $\phi(u)$ is convex, then $k_{0}<k_{1}<k_{2}<\cdots<k_{N}<\infty$ and $0>c_{1}>c_{2}>\cdots>c_{N}$. We note that $b_{1}, b_{2}, \ldots, b_{N}$ can be determined from $c_{1}, c_{2}, \ldots, c_{N}$ by the continuity of the function

$$
b_{i}=-\frac{c_{i}-c_{i-1}}{k_{i}-k_{i-1}} \quad\left(c_{0}=0\right)
$$

and vise versa. Fig. 1 plots a piecewise linear concave function with four bends.

\section{Describing the System Under SeCtor Condition With SATURATED LINEAR SYSTEMS}

Lemma 1 transforms the problem of verifying the absolute contractive invariance of a level set into one of verifying the contractive invariance of the level set under individual vertex functions. This simplifies the problem to some degree but there is still no solution even if each component of the vertex functions is piecewise linear. The only situation that we are able to address is where each component of the vertex functions is a piecewise linear function with only one bend over $[0, \infty)$. In this case, every vertex function can be decomposed into a linear term and a standard saturation function and the corresponding vertex system is a saturated linear system, for which a set of analysis tools have been recently developed (see, e.g., [16] and [18]-[20]). In this section, we use an array of saturation functions to describe a piecewise linear sector. By doing this, we transform the absolute stability problem into the stability analysis of an array of systems with saturation nonlinearities.

Let us first examine a scalar concave/convex piecewise linear function. The following lemma establishes a connection between a piecewise linear function and an array of saturation functions. 


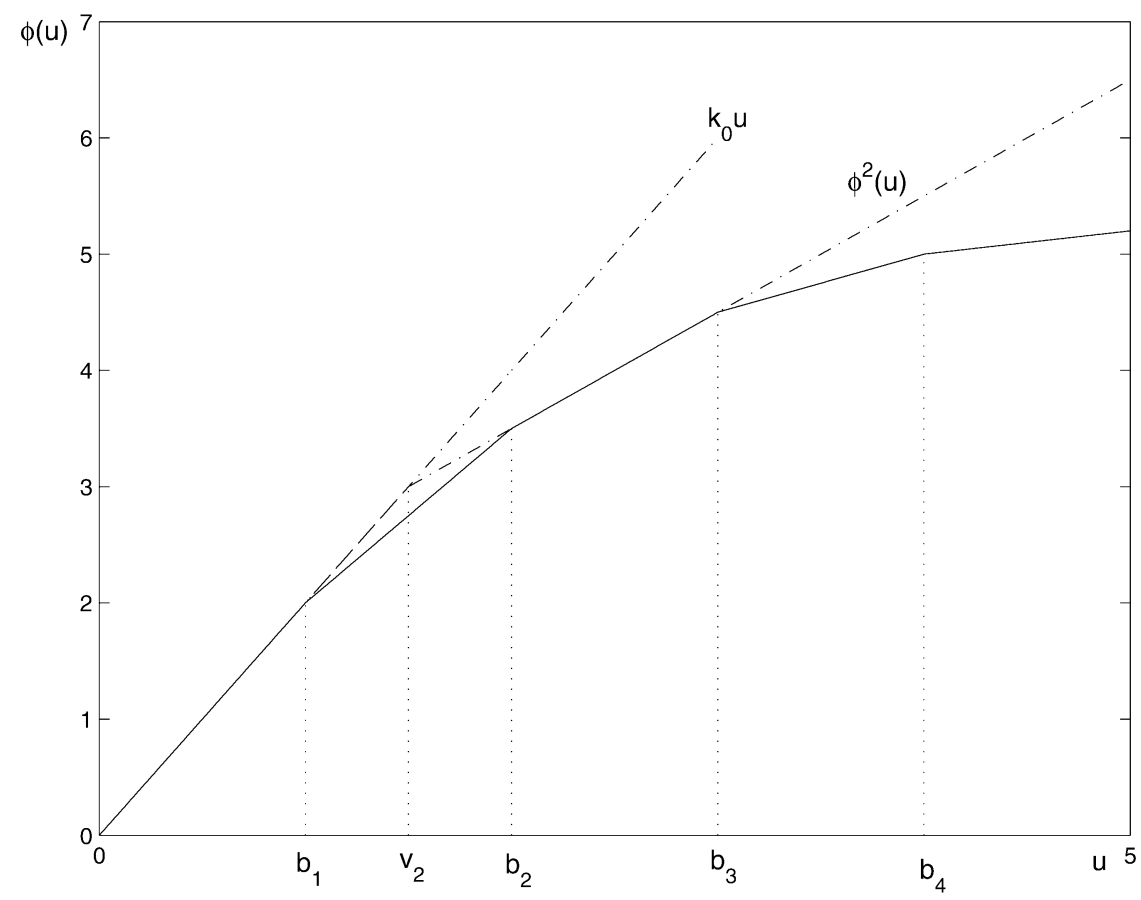

Fig. 1. Illustration for the proof of Lemma 2.

Lemma 2: Consider a piecewise linear concave/convex function

$$
\phi(u)= \begin{cases}k_{0} u, & \text { if } u \in\left[0, b_{1}\right] \\ k_{1} u+c_{1}, & \text { if } u \in\left(b_{1}, b_{2}\right] \\ \vdots & \\ k_{N} u+c_{N}, & \text { if } u \in\left(b_{N}, \infty\right) .\end{cases}
$$

For $j \in I[1, N]$, define

$$
\phi^{j}(u):=k_{j} u+c_{j} \operatorname{sat}\left(\frac{k_{0}-k_{j}}{c_{j}} u\right) .
$$

Then

$$
\begin{aligned}
\phi^{j}(u) & \in \operatorname{co}\left\{k_{0} u, \phi(u)\right\} \\
\phi(u) & \in \operatorname{co}\left\{\phi^{j}(u): j \in I[1, N]\right\} .
\end{aligned}
$$

Moreover, if $\phi$ is concave, then for $u \geq 0$

$\phi(u) \leq \phi^{j}(u) \leq k_{0} u, \quad \phi(u)=\min \left\{\phi^{j}(u): j \in I[1, N]\right\}$.

If $\phi$ is convex, then for $u \geq 0$

$\phi(u) \geq \phi^{j}(u) \geq k_{0} u, \quad \phi(u)=\max \left\{\phi^{j}(u): j \in I[1, N]\right\}$.

Proof: We only give the proof for the case where $\phi(u)$ is concave. The case where $\phi(u)$ is convex is similar. The proof is illustrated in Fig. 1, where the piecewise linear function in solid line is $\phi(u)$. Also plotted in the figure are $\phi^{2}(u)$ and the straight line $k_{0} u$, both in dashed-dotted lines.

Let $v_{j}=\left(c_{j} /\left(k_{0}-k_{j}\right)\right)$. Then $\left(v_{j}, k_{0} v_{j}\right)$ is the intersection of the straight line $v=k_{0} u$ with the other straight line obtained by extending the $(j+1)$ th section of $\phi(u)$ to the left (see Fig. 1). It is clear that $0<v_{j} \leq b_{j}\left(v_{1}=b_{1}\right)$ and

$$
\phi^{j}(u)= \begin{cases}k_{0} u, & \text { if } u \in\left[0, v_{j}\right] \\ k_{j} u+c_{j}, & \text { if } u \in\left(v_{j}, \infty\right) .\end{cases}
$$

It follows that

$$
\phi(u)= \begin{cases}\phi^{1}(u), & \text { if } u \in\left[0, b_{2}\right] \\ \vdots & \\ \phi^{j}(u), & \text { if } u \in\left(b_{j}, b_{j+1}\right] \\ \vdots & \\ \phi^{N}(u), & \text { if } u \in\left(b_{N}, \infty\right) .\end{cases}
$$

Hence

$$
\phi(u) \in \operatorname{co}\left\{\phi^{j}(u): j \in I[1, N]\right\} \quad \forall u \in \mathbf{R} .
$$

Since $\phi$ is concave, we have (see Fig. 1)

$$
k_{j} u+c_{j} \geq \phi(u), \quad k_{0} u \geq \phi(u) \quad \forall u \geq 0 .
$$

It follows that

$$
\phi(u) \leq \phi^{j}(u) \leq k_{0} u \quad \forall u \geq 0, j \in I[1, N]
$$

which implies (10). From (14), we have

$$
\phi(u) \geq \min \left\{\phi^{j}(u): j \in I[1, N]\right\} .
$$

From (15), we have

$$
\phi(u) \leq \min \left\{\phi^{j}(u): j \in I[1, N]\right\} .
$$

Thus, (12) is verified.

Here, we note that relations equivalent to (10) and (11) were contained in the proof of [13, Th. 2]. Applying Lemma 2, we can 

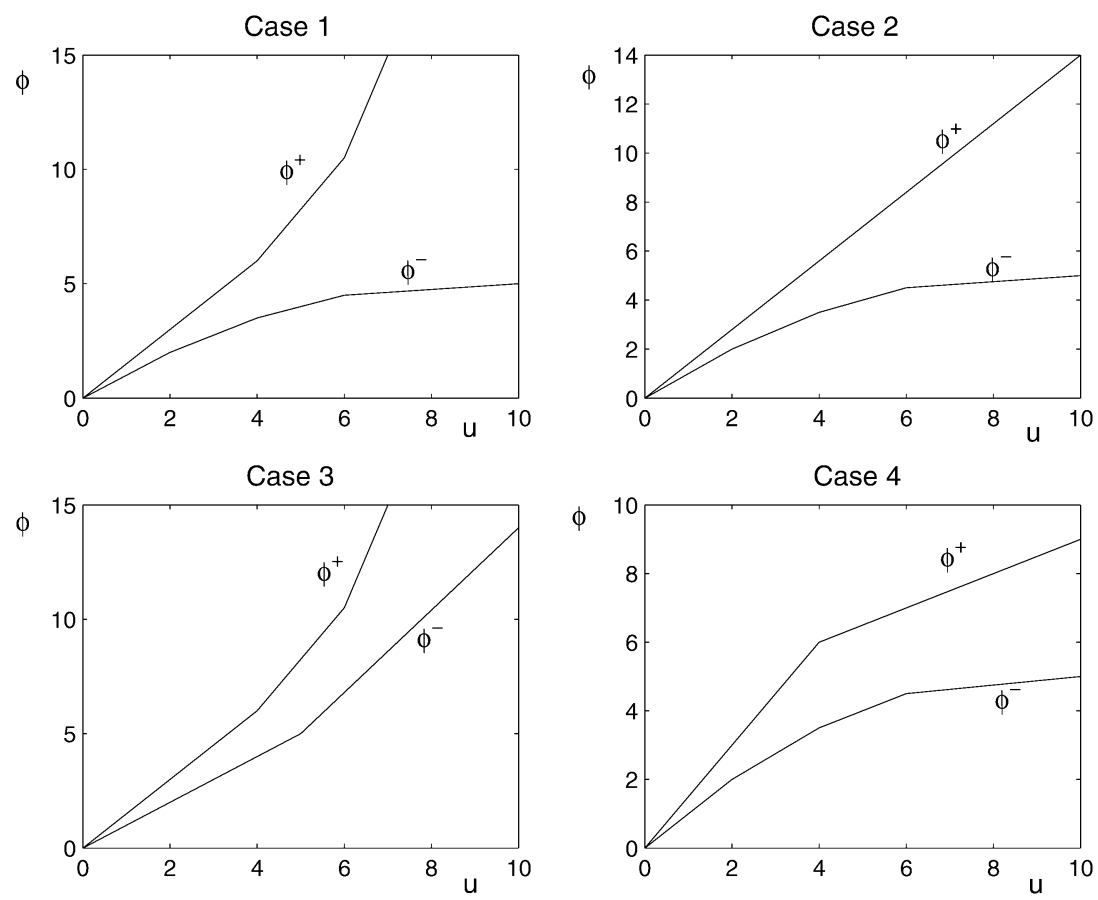

Fig. 2. Four cases of Lemma 3: $\bar{\phi}=\phi^{+}, \breve{\phi}=\phi^{-}$.

use a new set of vertex functions to replace the original piecewise linear vertex functions. The new set of vertex functions have components of the form $\phi^{j}$ as defined in (9). Let us first consider the scalar case where $\phi(\cdot, \cdot): \mathbf{R} \times \mathbf{Z} \rightarrow \mathbf{R}$ belongs to the sector $\operatorname{co}\{\bar{\phi}, \breve{\phi}\}$ with $\bar{\phi}$ and $\breve{\phi}$ being piecewise linear concave/convex functions

$$
\bar{\phi}(u)= \begin{cases}k_{01} u, & \text { if } u \in\left[0, b_{11}\right] \\ k_{11} u+c_{11}, & \text { if } u \in\left(b_{11}, b_{21}\right] \\ \vdots & \\ k_{N_{1} 1} u+c_{N_{1} 1}, & \text { if } u \in\left(b_{N_{1}}, \infty\right)\end{cases}
$$

and

$$
\breve{\phi}(u)= \begin{cases}k_{02} u, & \text { if } u \in\left[0, b_{12}\right] \\ k_{12} u+c_{12}, & \text { if } u \in\left(b_{12}, b_{22}\right] \\ \vdots & \\ k_{N_{2} 2} u+c_{N_{2} 2}, & \text { if } u \in\left(b_{N_{2}}, \infty\right) .\end{cases}
$$

Define

$$
\begin{aligned}
\bar{\phi}^{\ell}(u) & :=k_{\ell 1} u+c_{\ell 1} \operatorname{sat}\left(\frac{k_{01}-k_{\ell 1}}{c_{\ell 1}} u\right) \\
\ell & \in I\left[1, N_{1}\right] \\
\breve{\phi}^{j}(u) & :=k_{j 2} u+c_{j 2} \operatorname{sat}\left(\frac{k_{02}-k_{j 2}}{c_{j 2}} u\right) \\
j & \in I\left[1, N_{2}\right] .
\end{aligned}
$$

Then, by (12) and (13) of Lemma 2

$$
\operatorname{co}\{\bar{\phi}, \breve{\phi}\} \subset \operatorname{co}\left\{\bar{\phi}^{\ell}, \breve{\phi}^{j}: \ell \in I\left[1, N_{1}\right], j \in I\left[1, N_{2}\right]\right\} .
$$

Note that the sector on the right-hand side has vertex functions as the sum of linear functions and saturation functions.

We intend to use the sector on the right hand side of (20) to replace the one on the left-hand side so that we can use stability analysis tools available for systems with saturation nonlinearities. However, the difference between the two sectors may introduce conservatism. The following lemma lists several cases where the two sectors are the same.

Lemma 3: Assume that $\bar{\phi}(u) \geq \breve{\phi}(u)$ for $u \geq 0$. For the following cases:

$$
\operatorname{co}\{\bar{\phi}, \breve{\phi}\}=\operatorname{co}\left\{\bar{\phi}^{\ell}, \breve{\phi}^{j}: \ell \in I\left[1, N_{1}\right], j \in I\left[1, N_{2}\right]\right\} .
$$

1) $\bar{\phi}$ is convex and $\breve{\phi}$ is concave.

2) $\bar{\phi}$ or $\breve{\phi}$ is linear.

3) Both $\bar{\phi}$ and $\breve{\phi}$ are convex, $\breve{\phi}$ has only one bend and $\breve{\phi} \leq \bar{\phi}^{\ell}$, for all $\ell \in I\left[1, N_{1}\right]$.

4) Both $\bar{\phi}$ and $\breve{\phi}$ are concave, $\bar{\phi}$ has only one bend and $\bar{\phi} \geq \breve{\phi}^{j}$, for all $j \in I\left[1, N_{2}\right]$.

The four cases in Lemma 3 are plotted in Fig. 2.

Proof: Let $\bar{\phi}$ and $\breve{\phi}$ be in the form of (16) and (17). Then, $k_{01} \geq k_{02}$. For Case 1), it follows from (12) and (13) of Lemma 2 that for all $u \geq 0$

$$
\begin{aligned}
\bar{\phi}(u) & \geq \bar{\phi}^{\ell}(u) \geq k_{01} u \geq k_{02} u \geq \breve{\phi}^{j}(u) \geq \breve{\phi}(u) \\
\quad \ell & \in I\left[1, N_{1}\right], \quad j \in I\left[1, N_{2}\right] .
\end{aligned}
$$

Hence, we have

$$
\bar{\phi}^{\ell}, \breve{\phi}^{j} \in \operatorname{co}\{\bar{\phi}, \breve{\phi}\} \quad \forall \ell \in I\left[1, N_{1}\right], j \in I\left[1, N_{2}\right]
$$

and (21) is obtained.

For Case 2), if $\bar{\phi}$ is linear, then $N_{1}=1$ and

$$
\begin{aligned}
\bar{\phi}(u) & =\bar{\phi}^{\ell}(u)=k_{01} u \geq k_{02} u \geq \breve{\phi}^{j}(u) \geq \breve{\phi}(u) \\
\ell & \in I\left[1, N_{1}\right], \quad j \in I\left[1, N_{2}\right]
\end{aligned}
$$


and we also obtain (23) and (21). If $\breve{\phi}$ is linear, the argument is similar.

For Case 3), we have $N_{2}=1$ and

$$
\begin{aligned}
\bar{\phi}(u) & \geq \bar{\phi}^{\ell}(u) \geq \breve{\phi}^{j}(u)=\breve{\phi}(u) \\
\ell & \in I\left[1, N_{1}\right], \quad j \in I\left[1, N_{2}\right] .
\end{aligned}
$$

For Case 4), we have $N_{1}=1$ and

$$
\begin{aligned}
\bar{\phi}(u) & =\bar{\phi}^{\ell}(u) \geq \breve{\phi}^{j}(u) \\
\ell & \geq \breve{\phi}(u) \\
& \in I\left[1, N_{1}\right], \quad j \in I\left[1, N_{2}\right] .
\end{aligned}
$$

Both (25) and (26) imply (23) and, hence, (21).

Now, we consider the case where $m>1$. Assume that the $i$-th component $\phi_{i}\left(u_{i}, t\right)$ belongs to the sector $\operatorname{co}\left\{\bar{\phi}_{i}, \breve{\phi}_{i}\right\}$. Then, the piecewise linear sector for $\psi(u, t)$ is

$$
\operatorname{co}\left\{\psi^{j}: j \in I\left[1,2^{m}\right]\right\}
$$

where the $i$ th component of $\psi^{j}=\bar{\phi}_{i}$ or $\breve{\phi}_{i}$. Using Lemma 2 for each $i$, we can find $N_{i}$ functions of the form

$$
\phi_{i}^{\ell}\left(u_{i}\right)=\pi_{i \ell} u_{i}+\omega_{i \ell} \operatorname{sat}\left(\gamma_{i \ell} u_{i}\right), \quad \ell \in I\left[1, N_{i}\right]
$$

such that

$$
\operatorname{co}\left\{\bar{\phi}_{i}, \breve{\phi}_{i}\right\} \subset \operatorname{co}\left\{\phi_{i}^{\ell}: \ell \in I\left[1, N_{i}\right]\right\} .
$$

Hence, we have

$$
\begin{array}{r}
\operatorname{co}\left\{\psi^{j}: j \in\left[1,2^{m}\right]\right\} \subset \operatorname{co}\left\{\left[\begin{array}{llll}
\phi_{1}^{\ell_{1}} & \phi_{2}^{\ell_{2}} & \ldots & \phi_{m}^{\ell_{m}}
\end{array}\right]^{T}\right. \\
\left.: \ell_{i} \in I\left[1, N_{i}\right], i \in I[1, m]\right\},
\end{array}
$$

where the sector on the right hand side has $K:=N_{1} \times N_{2} \times \cdots \times$ $N_{m}$ vertices. If we assign a number $q \in I[1, K]$ to each vertex corresponding to $\left(\ell_{1}, \ell_{2}, \ldots, \ell_{m}\right)$, then each of the vertices has the form

$$
\begin{aligned}
& {\left[\begin{array}{llll}
\phi_{1}^{\ell_{1}}\left(u_{1}\right) & \phi_{2}^{\ell_{2}}\left(u_{2}\right) & \ldots & \phi_{m}^{\ell_{m}}\left(u_{m}\right)
\end{array}\right] T} \\
& =\Pi_{q} u+\Omega_{q} \operatorname{sat}\left(\Gamma_{q} u\right), \quad q \in I[1, K]
\end{aligned}
$$

where

$$
\begin{aligned}
& \Pi_{q}=\operatorname{diag}\left\{\pi_{1 \ell_{1}}, \pi_{2 \ell_{2}}, \ldots, \pi_{m \ell_{m}}\right\} \\
& \Omega_{q}=\operatorname{diag}\left\{\omega_{1 \ell_{1}}, \omega_{2 \ell_{2}}, \ldots, \omega_{m \ell_{m}}\right\} \\
& \Gamma_{q}=\operatorname{diag}\left\{\gamma_{1 \ell_{1}}, \gamma_{2 \ell_{2}}, \ldots, \gamma_{m \ell_{m}}\right\} .
\end{aligned}
$$

Replacing $\psi(u, t)$ of system (2) with a vertex function of the form (30), and letting $A_{q}=A+B \Pi_{q} F, B_{q}=B \Omega_{q}$ and $F_{q}=\Gamma_{q} F$, we obtain

$$
x^{+}=A_{q} x+B_{q} \operatorname{sat}\left(F_{q} x\right), \quad q \in I[1, K] .
$$

It then follows from Lemma 1 that if a convex level set is contractively invariant for each of the systems in (31), then it is absolutely contractively invariant for (2) under the sector condition $\phi_{i} \in \operatorname{co}\left\{\bar{\phi}_{i}, \breve{\phi}_{i}\right\}$ for each $i \in I[1, m]$.

\section{ANALYSIS OF SATURATED LiNEAR Systems With COMPOSITE QUADRATIC FUNCTIONS}

In Sections II and III, we converted the problem of verifying the ACI of a convex set into one of checking its contractive invariance for an array of saturated linear systems (31). In this section, we study the contractive invariance of the convex hull of a family of ellipsoids, which can be described as the level set of the composite quadratic Lyapunov function as introduced in [16]. In [16], such a level set was used to estimate the stability region for saturated continuous-time systems. In [20], the condition for such a level set to be contractively invariant was substantially relaxed through a stability condition for linear differential inclusions recently developed in [10]. In this section, we will derive a stability condition for linear difference inclusions by using the composite quadratic function and then establish the contractive invariance of its level set for saturated discrete-time systems. First, we give a brief review of the composite quadratic function and its properties.

\section{A. The Composite Quadratic Lyapunov Function}

Given a family of positive-definite matrices $Q_{j} \in \mathbf{R}^{n \times n}$, $Q_{j}=Q_{j}^{T}>0, j \in I[1, J]$. Let

$$
\Gamma=\left\{\gamma \in \mathbf{R}^{J}: \gamma_{1}+\gamma_{2}+\cdots+\gamma_{N}=1, \gamma_{j} \geq 0\right\} .
$$

The composite quadratic function is defined as

$$
V_{c}(x):=\min _{\gamma \in \Gamma} x^{T}\left(\sum_{j=1}^{J} \gamma_{j} Q_{j}\right)^{-1} x .
$$

For simplicity, we say that $V_{c}$ is composed from $Q_{j}, j \in I[1, J]$. When $J=1, V_{c}$ reduces to a quadratic function $x^{T} Q_{1}^{-1} x$. It is evident that $V_{c}$ is homogeneous of degree 2, i.e., $V_{c}(\alpha x)=$ $\alpha^{2} V_{c}(x)$. Also established in [10], [16] is that $V_{c}$ is convex and continuously differentiable.

Through convex analysis, it was also shown in [17] that

$$
\begin{aligned}
V_{c}(x) & =\max \left\{c^{T} x x^{T} c: c \in \cap_{j=1}^{J} \mathcal{E}\left(Q_{j}\right)\right\} \\
& =\max \left\{c^{T} x x^{T} c: c^{T} Q_{j} c \leq 1, j \in I[1, J]\right\}
\end{aligned}
$$

For $\rho>0$, the level set of $V_{c}$ is

$$
L_{V_{c}}(\rho):=\left\{x \in \mathbf{R}^{n}: V_{c}(x) \leq \rho\right\} .
$$

It is clear from the definition that

$$
V_{c}(x) \leq x^{T} Q_{j}^{-1} x \quad \forall j \in I[1, J] .
$$

Hence, $L_{V_{c}}(\rho) \supset \mathcal{E}\left(Q_{j}^{-1}, \rho\right)$ for all $\rho>0$ and $j \in I[1, J]$. Actually, from [16], we further have

$$
L_{V_{c}}(\rho)=\operatorname{co}\left\{\mathcal{E}\left(Q_{j}^{-1}, \rho\right): j \in I[1, J]\right\}
$$


where the right hand side denote the convex hull of the ellipsoids $\mathcal{E}\left(Q_{j}^{-1}, \rho\right): j \in I[1, J]$, i.e.,

$$
\begin{aligned}
\operatorname{co}\left\{\mathcal{E}\left(Q_{j}^{-1}, \rho\right), j\right. & \in I[1, J]\} \\
& =\left\{\sum_{j=1}^{J} \gamma_{j} x_{j}: x_{j} \in \mathcal{E}\left(Q_{j}^{-1}, \rho\right), \gamma \in \Gamma\right\} .
\end{aligned}
$$

For a compact convex set $S$, a point $x$ on the boundary of $S$ is called an extreme point if it cannot be represented as the convex combination of any other points in $S$. As a result, an extreme point of $L_{V_{c}}(\rho)$ must be on the boundary of $\mathcal{E}\left(Q_{j}^{-1}, \rho\right)$ for some $j \in I[1, J]$. In other words, if $x$ is an extreme point of $L_{V_{c}}(\rho)$, we must have $V_{c}(x)=x^{T} Q_{j}^{-1} x=\rho$ for some $j$.

\section{B. Stability Condition for Linear Difference Inclusions}

Consider the following linear difference inclusion:

$$
x^{+} \in \operatorname{co}\left\{A_{i} x: i \in I[1, N]\right\}
$$

where $A_{i} \in \mathbf{R}^{n \times n}, i \in I[1, N]$, are given. Let $V_{c}$ be composed from $Q_{j}=Q_{j}^{T}>0, j \in I[1, J]$. The following is a discretetime counterpart of the stability condition for linear differential inclusions in [10] and [20]. Similar to [10] and [20], a dual result can also be established by using a function conjugate to $V_{c}$. For simplicity, we only present the following result.

Theorem 1: If there exist $\lambda_{i j k} \geq 0, \sum_{k=1}^{J} \lambda_{i j k}=1, i \in$ $I[1, N], j, k \in I[1, J]$, such that

$A_{i}^{T}\left(\sum_{k=1}^{J} \lambda_{i j k} Q_{k}\right)^{-1} A_{i}<Q_{j}^{-1} \quad i \in I[1, N], j \in I[1, J]$

then $V_{c}\left(x^{+}\right)<V_{c}(x)$ for all $x \neq 0$. If $J=2$, then (36) is a necessary condition.

Proof: By homogeneity of the LDI and $V_{c}, V_{c}\left(x^{+}\right)<$ $V_{c}(x)$ for all $x \neq 0$ is equivalent to $V_{c}\left(x^{+}\right)<V_{c}(x)$ for all $x \in$ $L_{V_{c}}(1) \backslash\{0\}$. By the convexity of $V_{c}$ and linearity of $A_{i} x$, this is equivalent to $V_{c}\left(A_{i} x\right)<1$ for all $x \in L_{V_{c}}(1) \backslash\{0\}$ and each $i$. Again by the convexity of $V_{c}$ and linearity of $A_{i} x$, this is equivalent to $V_{c}\left(A_{i} x\right)<1$ for every extreme point of $L_{V_{c}}(1)$. If $x$ is an extreme point of $L_{V_{c}}(1)$, then we have $V_{c}(x)=x^{T} Q_{j}^{-1} x=1$ for some $j \in I[1, J]$. Therefore, $V_{c}\left(x^{+}\right)<V_{c}(x)$ for all $x \neq 0$ if and only if $V_{c}\left(A_{i} x\right)<1$ for all $x \in \mathcal{E}\left(Q_{j}^{-1}\right), j \in I[1, J]$.

Under (36) and by the definition of $V_{c}$, for $x \in \mathcal{E}\left(Q_{j}^{-1}\right) \backslash\{0\}$ we have

$$
V_{c}\left(A_{i} x\right) \leq x^{T} A_{i}^{T}\left(\sum_{k=1}^{J} \lambda_{i j k} Q_{k}\right)^{-1} A_{i} x<x^{T} Q_{j}^{-1} x \leq 1
$$

which confirms the sufficiency of the condition.

Before proving the necessity of the condition for the case $J=$ 2 , we need to show the following result.

Claim 1: Given $R=R^{T}, R_{1}=R_{1}^{T}, R_{2}=R_{2}^{T} \in \mathbf{R}^{n \times n}$. We have

$$
c^{T} R c<\max \left\{c^{T} R_{1} c, c^{T} R_{2} c\right\} \quad \forall c \in \mathbf{R}^{n} \backslash\{0\}
$$

if and only if there exists $\alpha \in$ [1] such that $R<\alpha R_{1}+(1-$ $\alpha) R_{2}$.

The "if" part is obvious. We show "only if." We can break (37) into two inequalities

$$
\begin{array}{ll}
c^{T} R c<c^{T} R_{1} c, & \text { if } c^{T}\left(R_{2}-R_{1}\right) c \leq 0 \\
c^{T} R c<c^{T} R_{2} c, & \text { if } c^{T}\left(R_{1}-R_{2}\right) c \leq 0 .
\end{array}
$$

By $\mathcal{S}$-procedure (e.g., see [4]), this implies the existence of $\alpha_{1}, \alpha_{2} \geq 0$ such that

$$
R<R_{1}+\alpha_{1}\left(R_{2}-R_{1}\right), \quad R<R_{2}+\alpha_{2}\left(R_{1}-R_{2}\right) .
$$

If either $\alpha_{1} \leq 1$ or $\alpha_{2} \leq 1$, then we are done. Now, suppose $\alpha_{1}, \alpha_{2}>1$. Let $\delta \in\left[\left(\alpha_{2}-1 /\left(\alpha_{1}+\alpha_{2}-1\right)\right),\left(\alpha_{2} /\left(\alpha_{1}+\right.\right.\right.$ $\left.\left.\left.\alpha_{2}-1\right)\right)\right]$. If we multiply the two inequalities in (40) with $\delta$ and $1-\delta$, respectively, and add them up, then we obtain $R<$ $\alpha R_{1}+(1-\alpha) R_{2}$ with $\alpha=\delta\left(1-\alpha_{1}\right)+(1-\delta) \alpha_{2} \in[0,1]$. This proves Claim 1 .

We now proceed to show the necessity of the condition for the case $J=2$. It suffices to verify that $V_{c}\left(A_{i} x\right)<1$ for all $x \in \mathcal{E}\left(Q_{j}^{-1}\right), j \in I[1,2]$ leads to (36) for every $i$ and $j$. By (33), $V_{c}\left(A_{i} x\right)<1$ for all $x \in \mathcal{E}\left(Q_{j}^{-1}\right)$ implies that

$$
\begin{aligned}
\max \left\{c^{T} A_{i} x x^{T} A_{i}^{T} c:\right. & c^{T} Q_{1} c \leq 1 \\
c^{T} Q_{2} c & \left.\leq 1, x^{T} Q_{j}^{-1} x \leq 1\right\}<1 .
\end{aligned}
$$

Observing that $\max \left\{c^{T} A_{i} x x^{T} A_{i}^{T} c: x^{T} Q_{j}^{-1} x \leq 1\right\}=$ $c^{T} A_{i} Q_{j} A_{i}^{T} c$, we have

$$
\max \left\{c^{T} A_{i} Q_{j} A_{i}^{T} c: c^{T} Q_{1} c \leq 1, c^{T} Q_{2} c \leq 1\right\}<1 .
$$

It can be verified routinely that (42) is equivalent to

$$
c^{T} A_{i} Q_{j} A_{i}^{T} c<\max \left\{c^{T} Q_{1} c, c^{T} Q_{2} c\right\} \quad \forall c \neq 0 .
$$

By Claim 1, this implies the existence of $\lambda_{i j k} \geq 0$, $k=1,2, \lambda_{i j 1}+\lambda_{i j 2}=1$, such that

$$
A_{i} Q_{j} A_{i}^{T}<\sum_{k=1}^{2} \lambda_{i j k} Q_{k} .
$$

By Schur complement, this is equivalent to

$$
\left[\begin{array}{cc}
\sum_{k=1}^{2} \lambda_{i j k} Q_{k} & A_{i} \\
A_{i}^{T} & Q_{j}^{-1}
\end{array}\right]>0
$$

and then to (36).

From the proof of Theorem 1, we see that condition (36) basically implies that $V_{c}$ is a common Lyapunov function for all the vertex systems $x^{+}=A_{i} x, i \in I[1, N]$. To determine if there 
exists such a $V_{c}$ for a given $J$, we need to solve $N \times J$ matrix inequalities in (36) with variables $Q_{j}, j \in I[1, J]$ and $\lambda_{i j k}$, $i \in I[1, N], j, k \in I[1, J]$. What we have done is to minimize the number $\beta$ such that there exist $Q_{j}$ 's and $\lambda_{i j k}$ 's satisfying

$$
\begin{aligned}
A_{i}^{T}\left(\sum_{k=1}^{J} \lambda_{i j k} Q_{k}\right)^{-1} A_{i} & <\beta Q_{j}^{-1} \\
i & \in I[1, N], \quad j \in I[1, J]
\end{aligned}
$$

or, equivalently

$$
\left[\begin{array}{cc}
\sum_{k=1}^{J} \lambda_{i j k} Q_{k} & A_{i} Q_{j} \\
Q_{j} A_{i}^{T} & \beta Q_{j}
\end{array}\right]>0, \quad i \in I[1, N], j \in I[1, J] .
$$

It fact, it can be shown similarly to the proof of Theorem 1 that if (47) is satisfied, then $V_{c}\left(x^{+}\right)<\beta V_{c}(x)$ for all $x \neq 0$. If the optimal $\beta \leq 1$, then the stability of the LDI is confirmed with $V_{c}$ composed from the solution $Q_{j}$ 's.

Remark 1: We note that (47) contains bilinear matrix inequalities (BMIs). A straightforward method to solve an optimization problem with BMI constraints is to break it up into a few problems with LMI constraints and solve them iteratively. However, this method does not work well on our particular problem. Some methods to address BMI constraints were developed in [3], [11], and [12], etc. In our computation, we adopt the path-following method in [12] and it turned out to be very effective. We first solve the problem of minimizing $\beta$ by assuming that all the $Q_{j}$ 's are equal to $Q$. This turns out to be a generalized eigenvalue problem. We then assign this optimal $Q$ to all $Q_{j}$ 's and randomly pick initial values for $\lambda_{i j k}$ so that $\sum_{k=1}^{J} \lambda_{i j k}=1$. The path-following algorithm is started with these $Q_{j}$ 's and $\lambda_{i j k}$ 's.

It is evident that as $J$ is increased, the optimal $\beta$ will decrease and the stability condition is less conservative. As expected, the computation effort will be more intense.

The following simple example shows that, when applied to stability analysis of LDIs, the composite quadratic functions with $J=2$ can improve substantially on what can be achieved with quadratic functions.

Example 1: Consider the following LDI:

$$
x^{+} \in \operatorname{co}\left\{A_{1} x, A_{2}(a) x\right\}
$$

where

$$
A_{1}=\left[\begin{array}{cc}
0.4 & -0.4 \\
0.4 & 0.4
\end{array}\right] \quad A_{2}(a)=\left[\begin{array}{cc}
0.4 & -\frac{0.4}{a} \\
0.4 a & 0.4
\end{array}\right], \quad a \geq 1 .
$$

The maximal $a$ such that there exists a common quadratic Lyapunov function for $A_{1}$ and $A_{2}(a)$ is $a_{1}=4.676$. By using Theorem 1 with $J=2$, the maximal $a$ that guarantees the existence of a common Lyapunov function $V_{c}$ is $a_{2}=7.546$.

\section{Invariant Level Set for Saturated Linear Systems}

Consider the saturated system

$$
x^{+}=A x+B \operatorname{sat}(F x)
$$

where $A \in \mathbf{R}^{n \times n}, B \in \mathbf{R}^{n \times m}$ and $F \in \mathbf{R}^{m \times n}$. We will use LDIs to describe this system within a local region of state-space. This description is made possible with a tool from [18] and [19].

Consider the set of $m \times m$ diagonal matrices whose diagonal elements are either 1 or 0 . There are $2^{m}$ such matrices and we label them as $D_{i}, i \in I\left[1,2^{m}\right]$. Denote $D_{i}^{-}=I-D_{i}$. Given two vectors $u, v \in \mathbf{R}^{m}$

$$
\left\{D_{i} u+D_{i}^{-} v: i \in\left[1,2^{m}\right]\right\}
$$

is the set of vectors obtained by choosing some elements from $u$ and the rest from $v$.

Lemma 4: [19] Let $H \in \mathbf{R}^{m \times n}$ be given. Then, for all $x \in$ $\mathcal{L}(h)$

$$
\operatorname{sat}(F x) \in \operatorname{co}\left\{\left(D_{i} F+D_{i}^{-} H\right) x: i \in I\left[1,2^{m}\right]\right\} .
$$

Let $h_{\ell}$ be the $\ell$ th row of $H$. Given $Q=Q^{T}>0 . \mathcal{E}\left(Q^{-1}\right) \subset$ $\mathcal{L}(H)$ if and only if $h_{\ell} Q h_{\ell}^{T} \leq 1$ for all $\ell \in I[1, m]$.

Let $Q_{j}=Q_{j}^{T}, j \in I[1, j]$ be positive-definite matrices and let $V_{c}$ be defined as in (32). The following theorem gives a sufficient condition for the contractive invariance of $L_{V_{c}}$ (1) for (48).

Theorem 2: If there exist an $H \in \mathbf{R}^{m \times n}$ and $\lambda_{i j k} \geq 0$, $\sum_{k=1}^{J} \lambda_{i j k}=1, i \in I\left[1,2^{m}\right], j, k \in I[1, J]$, such that (49) and (50), as shown at the bottom of the page, hold, where $h_{\ell}$ is the $\ell$ th row of $H$. Then, $L_{V_{c}}(1)$ is contractively invariant, i.e.,

$$
V_{c}\left(x^{+}\right)<V_{c}(x) \quad \forall x \in L_{V_{c}}(1) \backslash\{0\} .
$$

Proof: By Lemma 4, (50) implies that $\mathcal{E}\left(Q_{j}^{-1}\right) \subset \mathcal{L}(H)$ for all $j \in I[1, J]$. Since $L_{V_{c}}(1)$ is the convex hull of $\mathcal{E}\left(Q_{j}^{-1}\right)$, $j \in I[1, J]$, it follows that $L_{V_{c}}(1) \subset \mathcal{L}(H)$. Also, by Lemma 4 , we have

$$
\begin{aligned}
& \operatorname{sat}(F x) \in \operatorname{co}\left\{\left(D_{i} F+D_{i}^{-} H\right) x: i \in I\left[1,2^{m}\right]\right\} \\
& \forall x \in L_{V_{c}}(1) .
\end{aligned}
$$

If we let $A_{i}=A+B\left(D_{i} F+D_{i}^{-} H\right)$, then for all $x \in L_{V_{c}}(1)$

$$
x^{+}=A x+B \operatorname{sat}(F x) \in \operatorname{co}\left\{A_{i} x: i \in I\left[1,2^{m}\right]\right\} \text {. }
$$

By the Schur complement, (49) implies

$$
A_{i}^{T}\left(\sum_{k=1}^{J} \lambda_{i j k} Q_{k}\right)^{-1} A_{i}<Q_{j}^{-1}, \quad i \in I[1, N], j \in I[1, J] .
$$

Hence, by Theorem 1, we have

$$
V_{c}\left(x^{+}\right)<V_{c}(x) \quad \forall x \in L_{V_{c}}(1) \backslash\{0\} .
$$

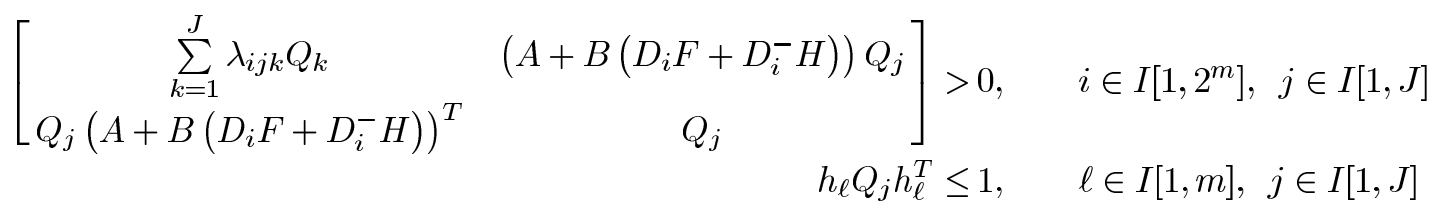


In Theorem 2, the condition for $L_{V_{c}}(1)$ to be contractively invariant involves $\mathcal{E}\left(Q_{j}^{-1}\right) \subset \mathcal{L}(H)$ for all $j \in I[1, J]$. For the special case $m=1$, we can use different $H$ for different $\mathcal{E}\left(Q_{j}^{-1}\right)$. By doing this, the condition for stability can be relaxed significantly.

Theorem 3: Assume that $m=1$. If there exist $H_{j} \in \mathbf{R}^{1 \times n}$ and $\alpha_{j k} \geq 0, \beta_{j k} \geq 0, \sum_{k=1}^{J} \alpha_{j k}=1, \sum_{k=1}^{J} \beta_{j k}=1$, and $j, k \in I[1, J]$ such that

$$
\begin{aligned}
& {\left[\begin{array}{cc}
\sum_{k=1}^{J} \alpha_{j k} Q_{k} & (A+B F) Q_{j} \\
\left.Q_{j}(A+B F)\right)^{T} & Q_{j}
\end{array}\right]>0, \quad j \in I[1, J]} \\
& {\left[\begin{array}{cc}
\sum_{k=1}^{J} \beta_{j k} Q_{k} & \left(A+B H_{j}\right) Q_{j} \\
\left.Q_{j}\left(A+B H_{j}\right)\right)^{T} & Q_{j}
\end{array}\right]>0, \quad j \in I[1, J]} \\
& H_{j} Q_{j} H_{j}^{T} \leq 1, \quad j \in I[1, J] .
\end{aligned}
$$

Then, $L_{V_{c}}(1)$ is contractively invariant.

To prove Theorem 3, we need a result which only holds for systems with one saturation component.

Lemma 5: Suppose $x_{0} \in \operatorname{co}\left\{x_{j}: j \in I\left[1, J_{0}\right]\right\}$ and $F x_{j} \geq$ 0 for all $j \in I\left[1, J_{0}\right]$. Then

$A x_{0}+B \operatorname{sat}\left(F x_{0}\right)$

$\in \operatorname{co}\left\{A x_{j}+B F x_{j}, A x_{j}+B \operatorname{sat}\left(F x_{j}\right): j \in I\left[1, J_{0}\right]\right\}$. $\mathbf{R}^{n}$

Proof: To prove (55), it suffices to show that for any $c \in$

$$
c^{T}\left(A x_{0}+B \operatorname{sat}\left(F x_{0}\right)\right) \in\left[\alpha_{\min }, \alpha_{\max }\right]
$$

where

$$
\begin{aligned}
& \alpha_{\min }=\min \left\{c^{T}\left(A x_{j}+B F x_{j}\right),\right. \\
& \left.c^{T}\left(A x_{j}+B \operatorname{sat}\left(F x_{j}\right)\right): j \in I\left[1, J_{0}\right]\right\} \\
& \alpha_{\max }=\max \left\{c^{T}\left(A x_{j}+B F x_{j}\right),\right. \\
& \left.c^{T}\left(A x_{j}+B \operatorname{sat}\left(F x_{j}\right)\right): j \in I\left[1, J_{0}\right]\right\} .
\end{aligned}
$$

If $c^{T} B \geq 0$, then by the concavity of $\operatorname{sat}(u)$ for $u \geq 0$ and $F x_{0} \geq 0$, we have

$$
\begin{aligned}
& c^{T}\left(A x_{0}+B \operatorname{sat}\left(F x_{0}\right)\right) \\
& \quad \leq c^{T}\left(A x_{0}+B F x_{0}\right) \\
& \quad \leq \max \left\{c^{T}\left(A x_{j}+B F x_{j}\right): j \in I\left[1, J_{0}\right]\right\} \quad \leq \alpha_{\max }
\end{aligned}
$$
and

$$
\begin{aligned}
& c^{T}\left(A x_{0}+B \operatorname{sat}\left(F x_{0}\right)\right) \\
& \quad \geq \min \left\{c^{T}\left(A x_{j}+B \operatorname{sat}\left(F x_{j}\right)\right): j \in I\left[1, J_{0}\right]\right\} \geq \alpha_{\min } .
\end{aligned}
$$

Hence, we obtain (56).
If $c^{T} B \leq 0$, then by the convexity of $-\operatorname{sat}(u)$ for $u \geq 0$ and $F x_{0} \geq 0$

$$
\begin{aligned}
c^{T}\left(A x_{0}+B \operatorname{sat}\left(F x_{0}\right)\right) & \geq c^{T}\left(A x_{0}+B F x_{0}\right) \\
& \geq \min \left\{c^{T}\left(A x_{j}+B F x_{j}\right)\right. \\
& \left.\quad: j \in I\left[1, J_{0}\right]\right\} \\
& \geq \alpha_{\min }
\end{aligned}
$$

and

$$
\begin{gathered}
c^{T}\left(A x_{0}+B \operatorname{sat}\left(F x_{0}\right)\right) \leq \max \left\{c^{T}\left(A x_{j}+B \operatorname{sat}\left(F x_{j}\right)\right)\right. \\
\left.\quad: j \in I\left[1, J_{0}\right]\right\} \\
\leq \alpha_{\max } .
\end{gathered}
$$

We also have (56).

Proof of Theorem 3: By Schur complement, conditions (52) and (53) are equivalent to

$$
\begin{aligned}
(A+B F)^{T}\left(\sum_{k=1}^{J} \alpha_{j k} Q_{k}\right)^{-1}(A+B F) & <Q_{j}^{-1} \\
j & \in I[1, J](57) \\
\left(A+B H_{j}\right)^{T}\left(\sum_{k=1}^{J} \beta_{j k} Q_{k}\right)^{-1}\left(A+B H_{j}\right) & <Q_{j}^{-1} \\
j & \in I[1, J] .
\end{aligned}
$$

It follows that for each $\rho>0, j \in I[1, J]$

$$
\begin{aligned}
V_{c}(A x+B F x) \leq & x^{T}(A+B F)^{T}\left(\sum_{k=1}^{J} \alpha_{j k} Q_{k}\right)^{-1} \\
& \times(A+B F) x<x^{T} Q_{j}^{-1} x \\
\leq & \rho \quad \forall x \in \mathcal{E}\left(Q_{j}^{-1}, \rho\right) \backslash\{0\}
\end{aligned}
$$

and

$$
\begin{aligned}
\left.V_{c}\left(A x+B H_{j} x\right)\right) \leq & x^{T}\left(A+B H_{j}\right)^{T}\left(\sum_{k=1}^{J} \beta_{j k} Q_{k}\right)^{-1} \\
& \times\left(A+B H_{j}\right) x<x^{T} Q_{j}^{-1} x \\
\leq & \rho \quad \forall x \in \mathcal{E}\left(Q_{j}^{-1}, \rho\right) \backslash\{0\} .
\end{aligned}
$$

By (54), we have $\mathcal{E}\left(Q_{j}^{-1}\right) \subset \mathcal{L}\left(H_{j}\right)$. Hence, by Lemma 4 we have $A x+\operatorname{sat}(F x) \in\left\{(A+B F) x,\left(A+B H_{j}\right) x\right\}$ for all $x \in \mathcal{E}\left(Q_{j}^{-1}\right)$. It follows from (59), (60), and the convexity of $V_{c}$ that for each $\rho \in(0,1]$, we have

$$
\left.V_{c}(A x+B \operatorname{sat}(F x))\right)<\rho \quad \forall x \in \mathcal{E}\left(Q_{j}^{-1}, \rho\right) \backslash\{0\} .
$$

To prove the theorem, it suffices to show that for any $\rho \in$ $(0,1]$ and $x_{0} \in \partial L_{V_{c}}(\rho)$

$$
V_{c}\left(A x_{0}+B \operatorname{sat}\left(F x_{0}\right)\right)<V_{c}\left(x_{0}\right)=\rho .
$$

Here, we only prove the case where $\rho=1$. The proof for $\rho \in$ $(0,1)$ follows from same arguments. Hence, we need to show that for any $x_{0} \in \partial L_{V_{c}}(1)$

$$
V_{c}\left(A x_{0}+B \operatorname{sat}\left(F x_{0}\right)\right)<1 .
$$


Now, we consider an arbitrary $x_{0} \in \partial L_{V_{c}}(1)$. For simplicity, assume that $F x_{0} \geq 0$ (the proof for $F x_{0} \leq 0$ is similar). If $x_{0} \in \partial \mathcal{E}\left(Q_{j}^{-1}\right) \cap \partial L_{V_{c}}(1)$ for some $j \in I[1, J]$, then by (61)

$$
V_{c}\left(A x_{0}+B \operatorname{sat}\left(F x_{0}\right)\right)<1 .
$$

Now, we assume that $x_{0} \notin \partial \mathcal{E}\left(Q_{j}^{-1}\right)$ for any $j$. Since $L_{V_{c}}(1)$ is the convex hull of $\mathcal{E}\left(Q_{j}^{-1}, 1\right), j \in I[1, J]$, all the extreme points of $L_{V_{c}}(1)$ belong to the union of $\partial \mathcal{E}\left(Q_{j}^{-1}, 1\right)$, $j \in I[1, J]$. Hence, there exist an integer $J_{0} \leq J$, some vectors $x_{j} \in \partial \mathcal{E}\left(Q_{j}^{-1}\right), j \in I\left[1, J_{0}\right]$, such that

$$
x_{0} \in \operatorname{co}\left\{x_{j}: j \in I\left[1, J_{0}\right]\right\} .
$$

(Here, we have assumed for simplicity that $x_{0}$ is only related to the first $J_{0}$ ellipsoids. Otherwise, the ellipsoids can be reordered to meet this assumption.) From (61), we have

$$
V_{c}\left(A x_{j}+B \operatorname{sat}\left(F x_{j}\right)\right)<1, \quad j \in I\left[1, J_{0}\right] .
$$

and by (59)

$$
V_{c}\left(A x_{j}+B F x_{j}\right)<1, \quad j \in I\left[1, J_{0}\right] .
$$

We first consider the case where $F x_{j} \geq 0$ for all $j \in I\left[1, J_{0}\right]$. By Lemma 5, we have

$$
\begin{aligned}
& A x_{0}+B \operatorname{sat}\left(F x_{0}\right) \\
& \in \operatorname{co}\left\{A x_{j}+B F x_{j}, A x_{j}+B \operatorname{sat}\left(F x_{j}\right): j \in I\left[1, J_{0}\right]\right\} .
\end{aligned}
$$

Then, (63) follows from (64)-(66) and the convexity of $V_{c}$.

Next, we consider the case where $F x_{j}<0$ for some $j \in$ $I\left[1, J_{0}\right]$. Since $F x_{j} \geq 0$ does not hold for all $j \in I\left[1, J_{0}\right]$, we can get an intersection of the set $\operatorname{co}\left\{x_{1}, x_{2}, \ldots, x_{J_{0}}\right\}$ with the half space $F x \geq 0$. This intersection is also a polygon and can be denoted as $\operatorname{co}\left\{y_{1}, y_{2}, \ldots, y_{J_{1}}\right\}$. Since $F x_{0} \geq 0$, we have $x_{0} \in \operatorname{co}\left\{y_{1}, y_{2}, \ldots, y_{J_{1}}\right\}$ and by Lemma 5, we have

$$
\begin{aligned}
& A x_{0}+B \operatorname{sat}\left(F x_{0}\right) \\
& \in \operatorname{co}\left\{A y_{j}+B F y_{j}, A y_{j}+B \operatorname{sat}\left(F y_{j}\right): j \in I\left[1, J_{1}\right]\right\} .
\end{aligned}
$$

We note that some $y_{j}$ 's belong to $\left\{x_{1}, x_{2}, \ldots, x_{J_{0}}\right\}$, others are not. For those $y_{j} \notin\left\{x_{i}: i \in I\left[1, J_{0}\right]\right\}$, we must have $F y_{j}=0$ and $y_{j} \in \operatorname{co}\left\{x_{i}: i \in I\left[1, J_{0}\right]\right\}$. It follows from (65) and the convexity of $V_{c}$ that for these $y_{j}$, we also have

and

$$
V_{c}\left(A y_{j}+B F y_{j}\right)<1
$$

$$
V_{c}\left(A y_{j}+B \operatorname{sat}\left(F y_{j}\right)\right)=V_{c}\left(A y_{j}+B F y_{j}\right)<1 .
$$

In summary, we have

$$
\begin{gathered}
V_{c}\left(A y_{j}+B F y_{j}\right)<1 \quad V_{c}\left(A y_{j}+B \operatorname{sat}\left(F y_{j}\right)\right)<1 \\
j \in I\left[1, J_{1}\right] .
\end{gathered}
$$

Combining (67), (68), and the convexity of $V_{c}$, we obtain (63).

We finally consider the special case where both $m=1$ and $J=1$. In this case, $V_{c}$ reduces to a quadratic function $x^{T} Q^{-1} x$ and the level set $L_{V_{c}}(1)$ reduces to an ellipsoid $\mathcal{E}\left(Q^{-1}\right)$. Accordingly, the conditions in Theorem 3 can be transformed into LMIs by introducing the new variable $Y=H Q$. For continuous-time system, it was shown that the corresponding condition is necessary and sufficient for the contractive invariance of an ellipsoid [15]. The counterpart result for discrete-time system is established in Appendix A and summarized as follows.

Theorem 4: Assume $m=1$. Given $Q=Q^{T}>0$. The ellipsoid $\mathcal{E}\left(Q^{-1}\right)$ is contractively invariant for (48) if and only if there exists an $H \in \mathbf{R}^{1 \times n}$ such that

$$
\begin{aligned}
& {\left[\begin{array}{cc}
Q & (A+B F) Q \\
Q(A+B F)^{T} & Q
\end{array}\right]>0} \\
& {\left[\begin{array}{cc}
Q & (A+B H) Q \\
Q(A+B H)^{T} & Q
\end{array}\right]>0} \\
& H Q H^{T} \leq 1 \text {. }
\end{aligned}
$$

\section{Estimation of Stability Region With ACI LeVel Sets}

\section{A. Conditions for ACI Level Sets}

We return to the system with a sector condition

$$
x^{+}=A x+B \psi(F x, t)
$$

where $\psi_{i} \in \operatorname{co}\left\{\breve{\phi}_{i}, \bar{\phi}_{i}\right\}, i \in I[1, m]$, and $\bar{\phi}_{i}, \breve{\phi}_{i}$ are given piecewise linear concave/convex functions. We would like to estimate the stability region using ACI level sets of $V_{c}$ which is composed from $J$ matrices $Q_{j}=Q_{j}^{T}>0$.

Section III transforms the problem of verifying the absolute contractive invariance of a level set into one of verifying its contractive invariance for an array of saturated linear systems

$$
x^{+}=A_{q} x+B_{q} \operatorname{sat}\left(F_{q} x\right), \quad q \in I[1, K] .
$$

Conditions for the contractive invariance of a level set of $V_{c}$ for each of the above systems are presented in Theorem 2 for the general case. Putting all these conditions together, we obtain the condition of ACI for a level set $L_{V_{c}}(1)$ as follows.

Theorem 5: If there exist $H_{q} \in \mathbf{R}^{m \times n}$ and $\lambda_{q i j k} \geq 0$, $\sum_{k=1}^{J} \lambda_{q i j k}=1, q \in I[1, K], i \in I\left[1,2^{m}\right], j, k \in I[1, J]$, such that (74) and (75), as shown at the bottom of the page, hold, where $h_{q, \ell}$ is the $\ell$ th row of $H_{q}$. Then, $L_{V_{c}}(1)$ is absolutely contractively invariant.

In what follows, we consider the case $m=1$, when $B$ has only one column and $\psi$ is a scalar function. Let the two boundary functions $\bar{\phi}$ and $\breve{\phi}$ be given as in (16) and (17), where $\bar{\phi}$ has $N_{1}$ bends and $\phi$ has $N_{2}$ bends. So, we have $K=N_{1}+N_{2}$

$$
\left[\begin{array}{cc}
\sum_{k=1}^{J} \lambda_{q i j k} Q_{k} & \left(A_{q}+B_{q}\left(D_{i} F_{q}+D_{i}^{-} H_{q}\right)\right) Q_{j} \\
Q_{j}\left(A_{q}+B_{q}\left(D_{i} F_{q}+D_{i}^{-} H_{q}\right)\right)^{T} & Q_{j}
\end{array}\right]>0, \quad q \in I[1, K], \quad i \in I\left[1,2^{m}\right], j \in I[1, J]
$$

$$
h_{q, \ell} Q_{j} h_{q, \ell}^{T} \leq 1, \quad q \in I[1, K], \quad \ell \in I[1, m], \quad j \in I[1, N]
$$


saturated linear systems in (73). The matrices of each system is given as follows. For $q \in I\left[1, N_{1}\right]$

$$
A_{q}=A+k_{q 1} B F \quad B_{q}=c_{q 1} B \quad F_{q}=\frac{k_{01}-k_{q 1}}{c_{q 1}} F
$$

and for $q \in I\left[N_{1}+1, N_{1}+N_{2}\right]$

$$
\begin{aligned}
A_{q} & =A+k_{q-N_{1}, 2} B F, \quad B_{q}=c_{q-N_{1}, 2} B, \\
F_{q} & =\frac{k_{02}-k_{q-N_{1}, 2}}{c_{q-N_{1}, 2}} F .
\end{aligned}
$$

It is easy to verify that $A_{q}+B_{q} F_{q}=A+k_{01} B F$ for $q \in$ $I\left[1, N_{1}\right]$ and $A_{q}+B_{q} F_{q}=A+k_{02} B F$ for $q \in I\left[N_{1}+\right.$ $\left.1, N_{1}+N_{2}\right]$. Combining Lemma 1 and Theorem 3, we have the following result.

Theorem 6: Assume that $m=1$. If there exist $H_{q j} \in \mathbf{R}^{1 \times n}$ and $\alpha_{1 j k}, \alpha_{2 j k}, \beta_{q j k} \geq 0, \sum_{k=1}^{J} \alpha_{1 j k}=1, \sum_{k=1}^{J} \alpha_{2 j k}=1$, $\sum_{k=1}^{J} \beta_{q j k}=1, q \in I[1, K], j, k \in I[1, J]$ such that (76)-(79), as shown at the bottom of the page, hold. Then, $L_{V_{c}}(1)$ is absolutely contractively invariant.

We finally consider the special case where $m=1$ and $J=1$. For this case, the level sets of $V_{c}$ reduce to ellipsoids. Using Theorem 7, the necessary and sufficient condition for the ACI of an ellipsoid can be obtained.

Theorem 7: Assume that $m=1$. Given $Q=Q^{T}>0$. The ellipsoid $\mathcal{E}\left(Q^{-1}\right)$ is ACI if and only if there exist $H_{q} \in \mathbf{R}^{1 \times n}$, $q \in I[1, K]$ such that

$$
\begin{aligned}
& {\left[\begin{array}{cc}
Q & \left(A+k_{01} B F\right) Q \\
Q\left(A+k_{01} B F\right)^{T} & Q
\end{array}\right]>0} \\
& {\left[\begin{array}{cc}
Q & \left(A+k_{02} B F\right) Q \\
Q\left(A+k_{02} B F\right)^{T} & Q
\end{array}\right]>0} \\
& {\left[\begin{array}{cc}
Q & \left(A_{q}+B_{q} H_{q}\right) Q \\
Q\left(A_{q}+B_{q} H_{q}\right)^{T} & Q
\end{array}\right]>0, \quad q \in I[1, K]} \\
& H_{q} Q H_{q}^{T} \leq 1, \quad q \in I[1, K] .
\end{aligned}
$$

Proof: The sufficiency follows directly from applying Theorem 6 with $J=1$. We prove the necessity. The ACI of the ellipsoid implies that for each $\rho \in(0,1], \mathcal{E}\left(Q^{-1}, \rho\right)$ is contractively invariant for

$$
x^{+}=A x+B \bar{\phi}(F x) .
$$

When $\rho$ is sufficiently small, $\bar{\phi}(F x)=k_{01} F x$ for all $x \in$ $\mathcal{E}\left(Q^{-1}, \rho\right)$. Hence, $\mathcal{E}\left(Q^{-1}, \rho\right)$ must be contractively invariant for

$$
x^{+}=A x+k_{01} B F x .
$$

By linearity, $\mathcal{E}\left(Q^{-1}\right)$ must also be contractively invariant for (85). This proves (80). For $q \in I\left[1, N_{1}\right]$, we have

$$
x^{+}=A_{q} x+B_{q} \operatorname{sat}\left(F_{q} x\right)=A x+B \bar{\phi}^{q}(F x)
$$

where

$$
\bar{\phi}^{q}(u)=k_{q 1} u+c_{q 1} \operatorname{sat}\left(\frac{k_{01}-k_{q 1}}{c_{q 1}} u\right) .
$$

By Lemma 2, we have $\bar{\phi}^{q}(u) \in \operatorname{co}\left\{k_{01} u, \bar{\phi}(u)\right\}$. Hence, the contractive invariance of $\mathcal{E}\left(Q^{-1}\right)$ for (86) follows from its contractive invariance for both (84) and (85). Applying Theorem 7 to (86), there exists $H_{q}$ satisfying (82) and (83). Similar arguments can be applied to $q \in I\left[N_{1}+1, N_{1}+N_{2}\right]$ by using the contractive invariance of $\mathcal{E}\left(Q^{-1}\right)$ for $x^{+}=A x+B \breve{\phi}(F x)$.

\section{B. Optimization of ACI Level Sets}

We recall that the set $L_{V_{c}}(1)$ is characterized by the matrices $Q_{j}, j \in I[1, J]$. By the definition of $V_{c}$ and using Schur complement, we have

$$
L_{V_{c}}(1)=\left\{x \in \mathbf{R}^{n}: \exists \gamma \in \Gamma \text { s.t. }\left[\begin{array}{cc}
1 & x^{T} \\
x & \sum_{j=1}^{J} \gamma_{j} Q_{j}
\end{array}\right] \geq 0\right\} .
$$

To obtain better estimation of the stability region, we would like to determine an ACI level set $L_{V_{c}}(1)$ as large as possible. Typically, we are given a set of reference points $x_{p} \in \mathbf{R}^{n}$, $p \in I[1, N]$ and would like to determine an ACI set so that it contains $\alpha x_{p}, p \in I[1, N]$ with $\alpha$ as large as possible. When it specifies to the level sets of $V_{c}$, we would like to determine matrices $Q_{j}, j \in I[1, J]$ satisfying the conditions of Theorems 5-7 and further more, it satisfies

$$
\left[\begin{array}{cc}
1 & \alpha x_{p}^{T} \\
\alpha x_{p} & \sum_{j=1}^{J} \gamma_{p j} Q_{j}
\end{array}\right] \geq 0, \quad \gamma_{p j} \geq 0, \quad \sum_{j=1}^{J} \gamma_{p j}=1
$$

with $\alpha$ as large as possible. For the case $m=1$, this objective can be formulated (by applying Theorem 6) into the optimization problem shown in (87) at the bottom of the next page. Similar optimization problem can be formulated based on Theorem

$$
\begin{aligned}
& {\left[\begin{array}{cc}
\sum_{k=1}^{J} \alpha_{1 j k} Q_{k} & \left(A+k_{01} B F\right) Q_{j} \\
Q_{j}\left(A+k_{01} B F\right)^{T} & Q_{j}
\end{array}\right]>0, \quad j \in I[1, J]} \\
& {\left[\begin{array}{cc}
\sum_{k=1}^{J} \alpha_{2 j k} Q_{k} & \left(A+k_{02} B F\right) Q_{j} \\
Q_{j}\left(A+k_{02} B F\right)^{T} & Q_{j}
\end{array}\right]>0, \quad j \in I[1, J]} \\
& {\left[\begin{array}{cc}
\sum_{k=1}^{J} \beta_{q j k} Q_{k} & \left(A_{q}+B_{q} H_{q j}\right) Q_{j} \\
Q_{j}\left(A_{q}+B_{q} H_{q j}\right)^{T} & Q_{j}
\end{array}\right]>0, \quad q \in I[1, K], j \in I[1, J]} \\
& H_{q j} Q_{j} H_{q j}^{T} \leq 1, \quad q \in I[1, K], \quad j \in I[1, J]
\end{aligned}
$$


5 for the case $m>1$. To simplify computation, we can introduce new parameters $Y_{q j}:=H_{q j} Q_{j}, q \in I[1, K], j \in I[1, J]$. Then, items d) and e) in the optimization problem can be replaced with

$$
\begin{aligned}
& \text { d) }\left[\begin{array}{cc}
\sum_{k=1}^{J} \beta_{q j k} Q_{k} & A_{q} Q_{j}+B_{q} Y_{q j} \\
Q_{j} A_{q}^{T}+Y_{q j}^{T} B_{q}^{T} & Q_{j}
\end{array}\right]>0 \\
& q \in I[1, K], j \in I[1, J] \\
& \text { e) }\left[\begin{array}{cc}
1 & Y_{q j} \\
Y_{q j}^{T} & Q_{j}
\end{array}\right] \geq 0, \quad q \in I[1, K], \quad j \in I[1, J] .
\end{aligned}
$$

The resulting optimization problem has BMIs as constraints. In our computation, we again used the path-following method in [12] and it also turned out to be very effective. Before starting the path-following algorithm, we solved (87) under the assumption that all $Q_{j}$ 's are equal to $Q$. In this case, all the constraints are LMIs. We then assign this optimal $Q$ to each $Q_{j}$, pick random $\alpha_{1 j k}$ 's, $\alpha_{2 j k}$ 's $\beta_{q j k}$ 's and $\gamma_{p j}$ 's satisfying f) and $\mathrm{g}$ ), and then start the path-following algorithm. Based on our computational experience, all the initial values set up this way lead to the same final solutions.

We note that if we let $J=1 \mathrm{in}$ (87), then g) is gone and we obtain an optimization problem to maximize the ACI ellipsoids with respect to $x_{p}$ 's. In this case, all the constraints are LMIs. Similar LMI problem can be derived for the case $m>1$.

\section{Numerical Examples}

The following two examples illustrate that using ACI level sets of $V_{c}$ with $J=2$ to estimate the stability region can improve significantly on what can be achieved by using ACI ellipsoids.

Example 2: Consider a second order system with one nonlinear component

$$
x^{+}=A x+B \tan ^{-1}(F x)
$$

where

$$
A=\left[\begin{array}{cc}
0.8 & 0 \\
0 & 1.2
\end{array}\right] \quad B=\left[\begin{array}{c}
0.6 \\
-0.8
\end{array}\right] \quad F=\left[\begin{array}{ll}
0.5 & 1
\end{array}\right] .
$$

As in [13], we use the linear function $\psi_{1}(u)=u$ and a piecewise linear function $\psi_{2}$ to bound $\tan ^{-1}(u)$, where $\psi_{2}$ is obtained by connecting a finite number of points on $\tan ^{-1}(u)$ including the origin. Here, we select six points $\left(u, \tan ^{-1}(u)\right), u=$ $0,1,2,3,5,8$. The resulting piecewise linear function $\psi_{2}(u)$ has the form of (7) with $N=5$

$$
\begin{aligned}
& \left(k_{0}, k_{1}, k_{2}, k_{3}, k_{4}, k_{5}\right) \\
& \quad=(0.7845,0.3218,0.1419,0.0622,0.0243,0) \\
& \left(c_{1}, c_{2}, c_{3}, c_{4}, c_{5}\right) \\
& \quad=(0.4636,0.8234,1.0625,1.2517,1.4464) .
\end{aligned}
$$

We choose the reference point as $x_{0}=\left[\begin{array}{ll}0 & 1\end{array}\right]^{T}$.

We first consider quadratic functions $(J=1)$. The maximal $\alpha$ such that $\alpha x_{0}$ is inside an ACI ellipsoid is $\alpha_{1}=3.6009$.

Next, we consider $V_{c}$ which is composed from two quadratic functions $(J=2)$. The maximal $\alpha$ such that $\alpha x_{0}$ is inside an ACI $L_{V_{c}}(1)$ is $\alpha_{2}=5.0970$. (The maximal $\alpha$ such that $\alpha x_{0}$ is inside the true stability region is 5.693 , as detected by simulation). For verification, the two matrices defining the optimal $V_{c}$ is given as follows:

$$
\begin{aligned}
Q_{1} & =\left[\begin{array}{cc}
61.9296 & -20.4315 \\
-20.4315 & 25.3023
\end{array}\right] \\
Q_{2} & =\left[\begin{array}{cc}
26.7952 & 5.2779 \\
5.2779 & 26.1436
\end{array}\right] .
\end{aligned}
$$

The resulting invariant level sets by using different Lyapunov functions are compared in the left box of Fig. 3, where the outermost boundary is the optimal $L_{V_{c}}(1)$ for $x_{0}$. It can be seen that $L_{V_{c}}(1)$ is the convex hull of two ellipsoids (thin solid curve). The ellipsoids plotted in dashed lines are the maximal

$$
\begin{aligned}
& \begin{array}{l}
\sup _{Q_{j}, H_{q j}, \alpha_{1 j k}, \alpha_{2 j k}, \beta_{q j k}, \gamma_{p j}} \alpha \\
\text { s.t. } \quad \text { a) }\left[\begin{array}{cc}
1 & \alpha x_{p}^{T} \\
\alpha x_{p} & \sum_{j=1}^{J} \gamma_{p j} Q_{j}
\end{array}\right] \geq 0, \quad p \in I[1, N]
\end{array} \\
& \text { b) }\left[\begin{array}{cc}
\sum_{k=1}^{J} \alpha_{1 j k} Q_{k} & \left(A+k_{01} B F\right) Q_{j} \\
Q_{j}\left(A+k_{01} F\right)^{T} & Q_{j}
\end{array}\right]>0, \quad j \in I[1, J] \\
& \text { c) }\left[\begin{array}{cc}
\sum_{k=1}^{J} \alpha_{2 j k} Q_{k} & \left(A+k_{02} F\right) Q_{j} \\
Q_{j}\left(A+k_{02} F\right)^{T} & Q_{j}
\end{array}\right]>0, \quad j \in I[1, J] \\
& \text { d) }\left[\begin{array}{cc}
\sum_{k=1}^{J} \beta_{q j k} Q_{k} & \left(A_{q}+B_{q} H_{q j}\right) Q_{j} \\
Q_{j}\left(A_{q}+B_{q} H_{q j}\right)^{T} & Q_{j}
\end{array}\right]>0, \quad q \in I[1, K], j \in I[1, J] \\
& \text { e) } H_{q j} Q_{j} H_{q j}^{T} \leq 1, \quad q \in I[1, K], \quad j \in I[1, J] \\
& \text { f) } Q_{j}=Q_{j}^{T}>0, \quad \alpha_{1 j k}, \alpha_{2 j k}, \beta_{q j k}, \gamma_{p j} \geq 0, \quad p \in I[1, N], q \in I\left[1,2^{m}\right], j, k \in I[1, J] \\
& \text { g) } \sum_{k=1}^{J} \alpha_{1 j k}=1, \quad \sum_{k=1}^{J} \alpha_{2 j k}=1 \quad \sum_{k=1}^{J} \beta_{q j k}=1 \quad \sum_{j=1}^{J} \gamma_{p j}=1
\end{aligned}
$$




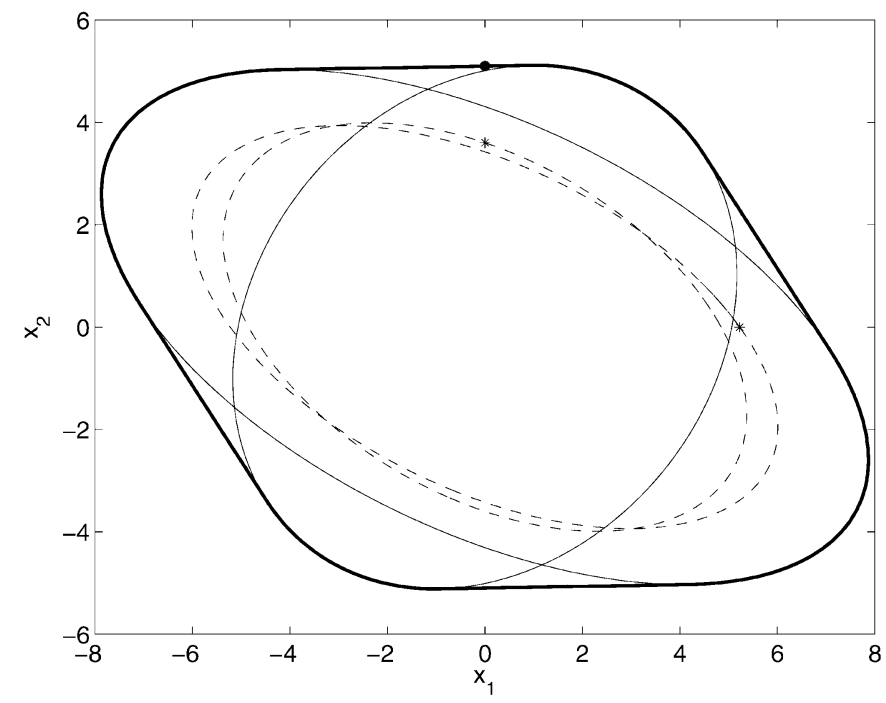

Fig. 3. (Left) ACI level sets. (Right) Next-step maps from $\partial L_{V_{c}}$.

ACI ellipsoids with respect to $x_{0}$ and $x_{1}=\left[\begin{array}{ll}1 & 0\end{array}\right]^{T}$, respectively. To verify that the resulting level set $L_{V_{c}}(1)$ is actually invariant, we plot the image of its boundary under the next-step map $x \mapsto A x+B \tan ^{-1}(F x)$ in the right box of Fig. 3 (see the thin solid lines). As a comparison, we also plot the images of the boundary under the map $x \mapsto A x+B \psi_{1}(F x)$ (dash-dotted) and the map $x \mapsto A x+B \psi_{2}(F x)$ (dashed), respectively. Since $\psi_{2}$ is very close to $\tan ^{-1}$, the dashed curve and the thin solid curve are very close.

Example 3: Consider a second order system with two nonlinear components

$$
x^{+}=A x+B \psi(F x)
$$

where

$$
\begin{aligned}
& A=\left[\begin{array}{cc}
1 & -0.04 \\
1 & 1
\end{array}\right] \quad B=\left[\begin{array}{cc}
0 & 1 \\
0.5 & 0.5
\end{array}\right] \\
& F=\left[\begin{array}{cc}
0.2727 & -0.2242 \\
-0.5097 & -0.1563
\end{array}\right]
\end{aligned}
$$

and $\psi_{1}\left(u_{1}\right) \in \operatorname{co}\left\{\operatorname{sat}\left(u_{1}\right), 2 u_{1}\right\}, \psi_{2}\left(u_{2}\right) \in \operatorname{co}\left\{\operatorname{sat}\left(u_{2}\right), 2 u_{2}\right\}$. The eigenvalues of $A$ are $1 \pm j 0.2$ and the eigenvalues of $A+B F$ are $0.65 \pm j 0.3841$. If we let $B_{1}$ and $B_{2}$ be the two columns of $B$ and let $F_{1}$ and $F_{2}$ be the two rows of $F$, we obtain four vertex systems

$$
\begin{aligned}
& x^{+}=(A+2 B F) x \\
& x^{+}=\left(A+2 B_{1} F_{1}\right) x+B_{2} \operatorname{sat}\left(F_{2} x\right) \\
& x^{+}=\left(A+2 B_{2} F_{2}\right) x+B_{1} \operatorname{sat}\left(F_{1} x\right) \\
& x^{+}=A x+B \operatorname{sat}(F x) .
\end{aligned}
$$

We take the reference point as $x_{0}=\left[\begin{array}{l}0 \\ 1\end{array}\right]$. We would like to determine an ACI set such that it contains $\alpha x_{0}$ with $\alpha$ as large as possible. If we optimize over all the ACI ellipsoids, the maximal $\alpha$ is $\alpha_{1}=10.1473$. If we optimize over ACI level sets of $V_{c}$ with $J=2$, the maximal $\alpha$ is $\alpha_{2}=19.0170$. Plotted in the left box of Fig. 4 are the optimized ACI ellipsoid (dashed line) and

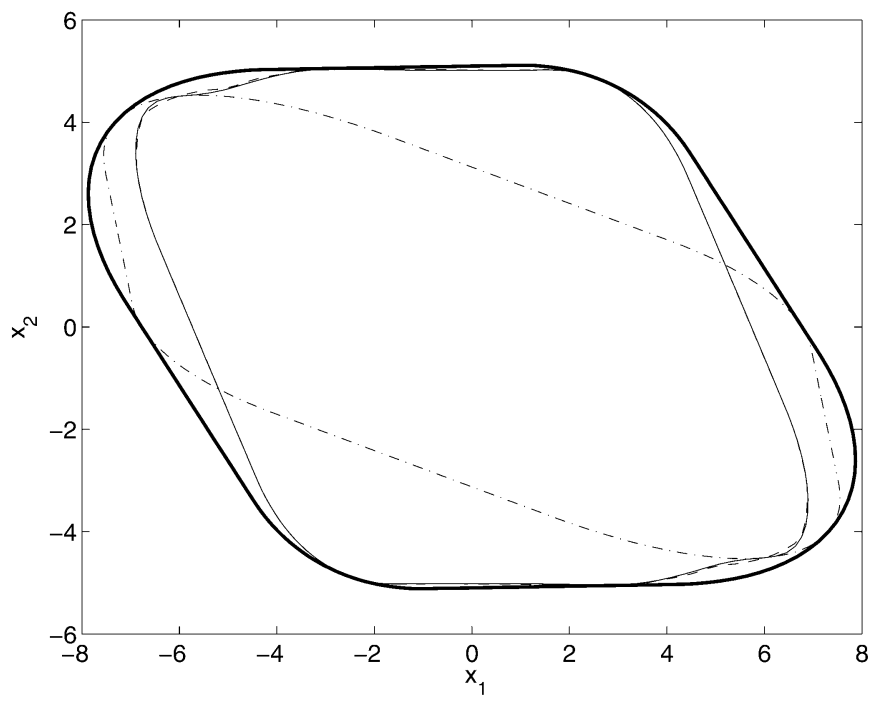

ACI $L_{V_{c}}(1)$ (thick solid line). Also plotted in this box in thin solid lines are the two ellipsoids $\mathcal{E}\left(Q_{1}^{-1}\right)$ and $\mathcal{E}\left(Q_{2}^{-1}\right)$ whose convex hull is $L_{V_{c}}(1)$. For verification, $Q_{1}$ and $Q_{2}$ are given as follows:

$$
\begin{aligned}
Q_{1} & =\left[\begin{array}{cc}
47.1064 & -92.8231 \\
-92.8231 & 356.9252
\end{array}\right] \\
Q_{2} & =\left[\begin{array}{cc}
40.9330 & -21.3000 \\
-21.3000 & 372.7298
\end{array}\right] .
\end{aligned}
$$

To demonstrate that this level set $L_{V_{c}}(1)$ is indeed absolutely contractively invariant, we computed the images of the boundary of $L_{V_{c}}(1)$ under the four next-step maps corresponding to the four vertex systems (88)-(91). These images are plotted with thin solid curves in the right box of Fig. 4. As can be clearly seen, all these images are within the level set $L_{V_{c}}(1)$.

As a matter of fact, we can also use $V_{c}$ to determine a region of instability using numerical simulation. The idea is to generate the "worst switching" among the four vertex systems so that at each point $x, x^{+}$is chosen as the one which maximizes $V_{c}$. By doing this, a diverging trajectory can be potentially produced. Fig. 5 plots a diverging trajectory produced this way (dashed-dotted line). The initial state is $\left[\begin{array}{ll}0 & 24.5\end{array}\right]^{T}$ (marked with “*”). A nearly closed trajectory is also produced under the worst switching strategy (see the curve in thin solid line). The region outside this nearly closed curve is deemed unstable. Also plotted in Fig. 5 is the same ACI level set as in Fig. 4. We notice that there is some gap between the estimated stability region (the ACI set) and the deemed region of instability. The true region of instability could include some points inside the nearly closed curve and the real stability region must be larger than the ACI set.

We note that with different switching strategies (possibly the worst case with respect to different Lyapunov functions), different regions of instability can be detected. The true region of instability for the system with sector condition will be the union of all these. 

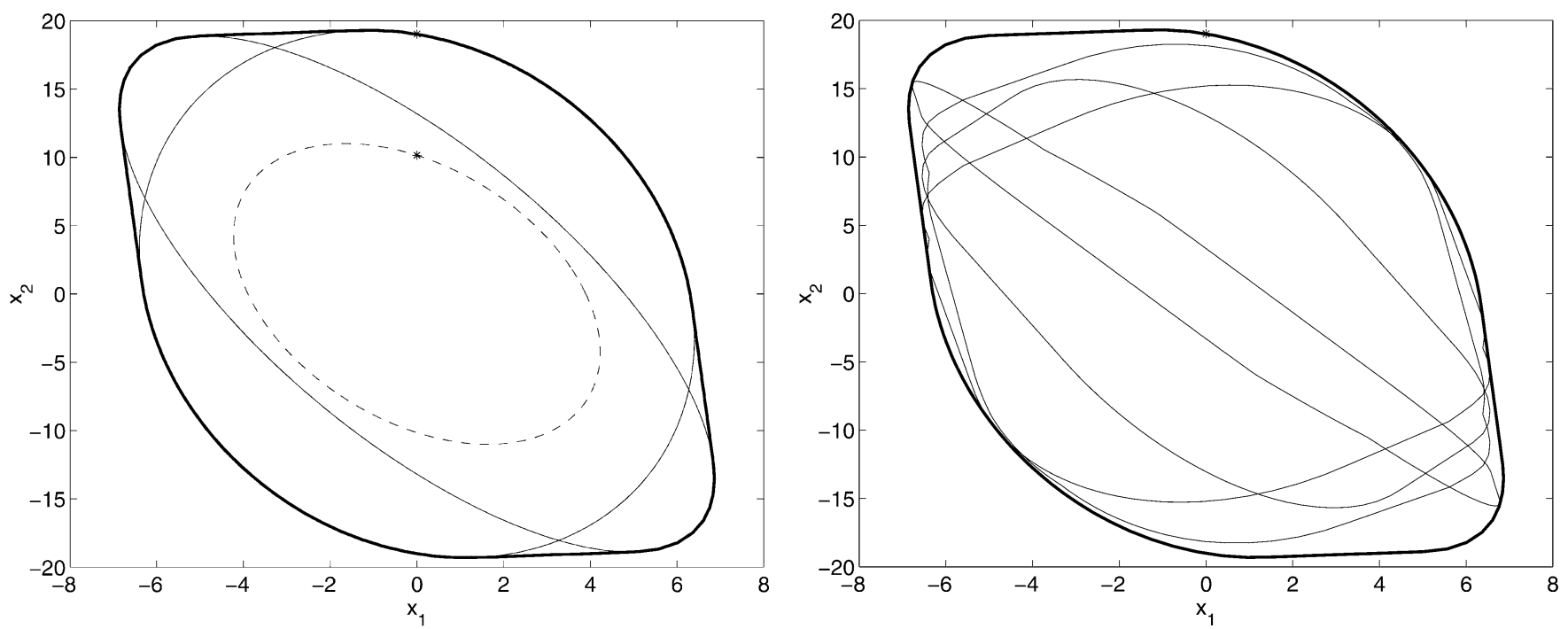

Fig. 4. (Left) ACI level sets. (Right) Next-step maps from $\partial L_{V_{c}}(1)$.

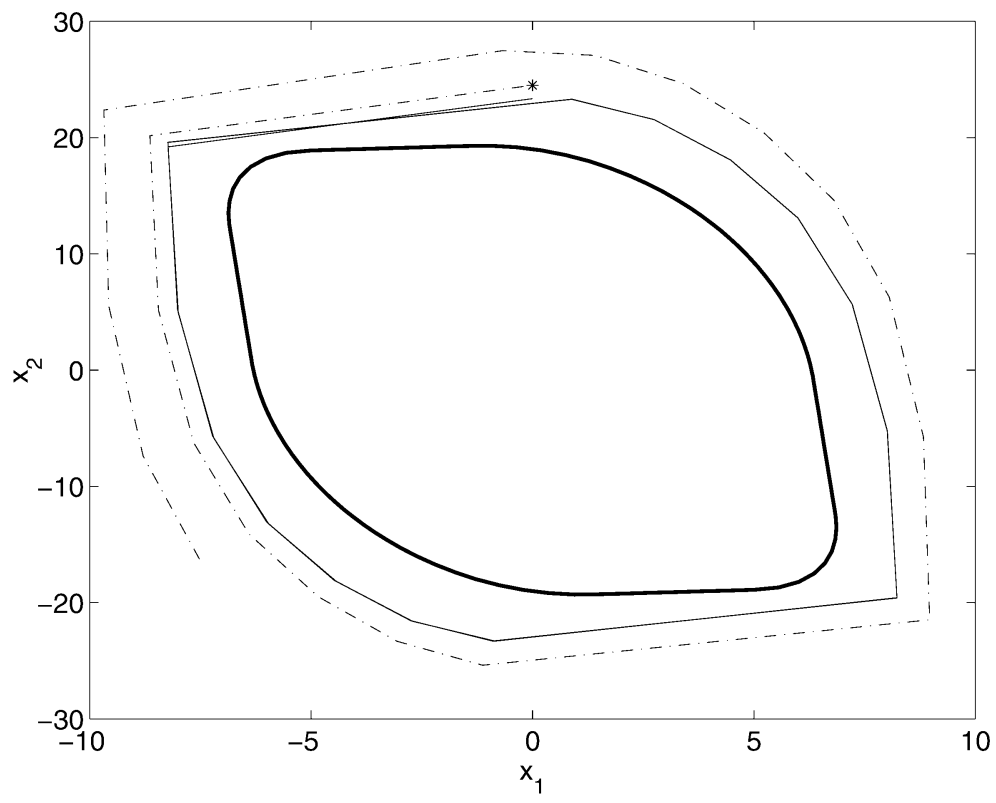

Fig. 5. Region of instability and a diverging trajectory.

\section{CONCLUSION}

We used composite quadratic Lyapunov function to enhance absolute stability analysis of systems with a piecewise linear sector condition. The composite quadratic Lyapunov function was introduced in our recent work [16] for stability analysis of saturated linear systems. Its properties have been further studied in [10], [17], and [20], and great potential has been demonstrated in the stability analysis of linear differential inclusions and saturated linear systems. Inspired by recent development in continuous-time systems, this paper first establishes stability conditions through composite quadratic Lyapunov functions for linear difference inclusions and saturated linear discrete-time systems. These results are used to establish conditions for absolute stability through a connection to saturated linear systems. With these conditions, the problem of estimating the stability region is formulated as optimization problems with bi- linear matrix inequalities which can be effectively solved with the path-following method in [12]. As illustrated by numerical examples, the stability region estimated by the composite quadratic Lyapunov function can be significantly larger than those by quadratic functions. The composite quadratic functions can also be used to generate the "worst switching" among a group of vertex systems for the purpose of detecting a potentially diverging trajectory.

\section{APPENDIX \\ Four EQUiVALENT STATEMENTS ABOUT INVARIANT ELLIPSOIDS}

Proposition 1: Given an ellipsoid $\mathcal{E}(P, \rho)$ and a row vector $F \in \mathbf{R}^{1 \times n}$. Assume that

$$
(A+B F)^{T} P(A+B F)-P<0 .
$$


The following four statements are equivalent.

a) The ellipsoid is contractively invariant for

$$
x^{+}=A x+B \operatorname{sat}(F x) .
$$

b) There exists a function $f: \mathbf{R} \rightarrow[-1,1]$ such that the ellipsoid is contractively invariant for

$$
x^{+}=A x+B f(x) .
$$

c) The ellipsoid is contractively invariant for

$x^{+}=A x+B \operatorname{sat}\left(F_{0} x\right) \quad F_{0}=-\left(B^{T} P B\right)^{-1} B^{T} P A$.

d) There exists an $H \in \mathbf{R}^{1 \times n}$ such that

$$
(A+B H)^{T} P(A+B H)-P<0
$$

and $\mathcal{E}(P, \rho) \subset \mathcal{L}(H)$.

We notice that Theorem 4 follows from the equivalence of a) and d). Let $Q=P / \rho$, then by using Schur complement it can be shown that (69) and (70) are equivalent to (92) and (96). Also, $H Q H^{T} \leq 1$ is equivalent to $\mathcal{E}(P, \rho) \subset \mathcal{L}(H)$.

Statement a) in Proposition 1 is about the invariance of an ellipsoid under a given feedback law. Statement b) is about the existence of a bounded feedback law to make the ellipsoid invariant. In statement $\mathrm{c})$, the feedback law $u=\operatorname{sat}\left(F_{0} x\right)$ maximizes the convergence rate with respect to the function $V(x)=$ $x^{T} P x$. Statement d) is about the existence of a feedback law linear inside the ellipsoid to make it invariant. There is no direct way to verify a), b), or c). The significance of Proposition 1 lies in the fact that all of them can be verified through d) which is numerically tractable.

The equivalence of a) and b) was established in [14] (see also [19]) and the equivalence of b) and c) was established in [14, p. 258]. It is also clear that d) implies b) and hence a) and c). In what follows, we will show that c) is equivalent to $d$ ).

Define

$\rho_{c}^{*}=\sup \{\rho>0: \mathcal{E}(P, \rho)$ is contractively invariant for $(95)\}$

and

$$
\begin{array}{ll} 
& \rho_{d}^{*}=\sup _{H} \rho \\
\text { s.t. } & (A+B H)^{T} P(A+B H)-P<0 \\
& H P^{-1} H^{T} \leq \frac{1}{\rho} .
\end{array}
$$

Note that $H P^{-1} H^{T} \leq(1 / \rho)$ is equivalent to $\mathcal{E}(P, \rho) \subset L(H)$. Since there exists an $\bar{H}$ satisfying (98) (actually, if we let $H=$ $F)$, the " $<$ " in (98) can be replaced with " $\leq$ ". Hence, the aforementioned optimization problem can also be written as

$$
\begin{gathered}
\left(\rho_{d}^{*}\right)^{-1}=\min _{H} H P^{-1} H^{T} \\
\text { s.t. }\left[\begin{array}{cc}
P & (A+B H)^{T} \\
A+B H & P^{-1}
\end{array}\right] \geq 0 .
\end{gathered}
$$

To prove Proposition 1, it suffices to show the following.

Lemma 6: $\rho_{c}^{*}=\rho_{d}^{*}$.
Proof: It is evident that $\rho_{c}^{*} \geq \rho_{d}^{*}$ (note that $\mathrm{b}$ ) and c) are equivalent). If there exists an $x \in \mathbf{R}^{n} \backslash\{0\}$ such that

$$
\left(A x+B \operatorname{sat}\left(F_{0} x\right)\right)^{T} P\left(A x+B \operatorname{sat}\left(F_{0} x\right)\right)-x^{T} P x=0
$$

then $\rho_{c}^{*}<\infty$ and

$$
\begin{array}{ll} 
& \rho_{c}^{*}=\min _{x \in \mathbf{R}^{n} \backslash\{0\}} x^{T} P x \\
\text { s.t. } & \left(A x+B \operatorname{sat}\left(F_{0} x\right)\right)^{T} P\left(A x+B \operatorname{sat}\left(F_{0} x\right)\right) \\
& -x^{T} P x=0 .
\end{array}
$$

To show $\rho_{c}^{*} \leq \rho_{d}^{*}$, it suffices to consider the case where $\rho_{d}^{*}<\infty$ and to construct an $x_{0} \in \mathbf{R}^{n}$ such that $x_{0}^{T} P x_{0}=\rho_{d}^{*}$ and $x_{0}$ satisfies (101).

For clarity, we divide the proof into three steps.

Step 1: Transformation of the optimization problem and normalization.

Denote

$$
W(H)=\left[\begin{array}{cc}
P & (A+B H)^{T} \\
A+B H & P^{-1}
\end{array}\right]
$$

and define

$$
\begin{aligned}
& S_{1}(\alpha):=\left\{H \in \mathbf{R}^{1 \times n}: W(H) \geq \alpha I\right\} \\
& S_{2}(\beta):=\left\{H \in \mathbf{R}^{1 \times n}: H P^{-1} H^{T} \leq \beta\right\} .
\end{aligned}
$$

Since $S_{1}(\alpha)$ is convex and $S_{2}(\beta)$ is strictly convex (any point between two distinct boundary points is not on the boundary), (100) implies that $S_{1}(0)$ and $S_{2}\left(\left(\rho_{d}^{*}\right)^{-1}\right)$ has a unique intersection $H_{*}$ with $\lambda_{\min }\left(W\left(H_{*}\right)\right)=0$, where $\lambda_{\min }(\cdot)$ denotes the smallest eigenvalue of a matrix. Furthermore, for all $H \in$ $S_{2}\left(\left(\rho_{d}^{*}\right)^{-1}\right) \backslash\left\{H_{*}\right\}$, we have $H \notin S_{1}(0)$, i.e., $\lambda_{\min }(W(H))<$ 0 . It follows that

$$
\begin{array}{ll} 
& 0=\max _{H} \lambda_{\min }(W(H)) \\
\text { s.t. } & H P^{-1} H^{T} \leq\left(\rho_{d}^{*}\right)^{-1}
\end{array}
$$

and the previous problem has a unique optimal solution $H_{*}$.

Without loss of generality and for simplicity, we assume that $P=I$ and $\rho_{d}^{*}=1$. Otherwise, we can make it so with a state transformation $\bar{x}=\left(P / \rho_{d}^{*}\right)^{(1 / 2)} x$. Thus, we have

$$
\begin{array}{ll} 
& 0=\max _{H} \lambda_{\min }(W(H)) \\
\text { s.t. } & H H^{T} \leq 1
\end{array}
$$

where

$$
W(H)=\left[\begin{array}{cc}
I & (A+B H)^{T} \\
A+B H & I
\end{array}\right] .
$$

Step 2: The eigenvector of $W\left(H_{*}\right)$.

Let $H_{*}$ be the unique optimal solution to (102). Then, $\lambda_{\min }\left(W\left(H_{*}\right)\right)=0$. Suppose that the multiplicity of the zero eigenvalue of $W\left(H_{*}\right)$ is $p$ and let $X \in \mathbf{R}^{n \times p}$ span the eigenspace of the zero eigenvalue. Then

$$
W\left(H_{*}\right) X=0 \text {. }
$$


We claim that $p=1$. Here, we need to resort to eigenvalue perturbation theory (see, e.g., [30]) to prove this claim. Let $H=$ $H_{*}+k \Delta H$, where $\Delta H$ represents the direction of perturbation. As $k$ is increased from $0, \lambda_{\min }\left(W\left(H_{*}+k \Delta H\right)\right.$ increases with a slope greater than $\varepsilon$ if and only if

$$
X^{T} \frac{\partial W\left(H_{*}+k \Delta H\right)}{\partial k} X>\varepsilon I .
$$

We note that, for a fixed $\Delta H$

$$
X^{T} \frac{\partial W\left(H_{*}+k \Delta H\right)}{\partial k} X=X^{T}\left[\begin{array}{cc}
0 & (B \Delta H)^{T} \\
B \Delta H & 0
\end{array}\right] X .
$$

Let $H_{0}$ be such that $W\left(H_{0}\right)>\eta I$ for some $\eta>0$ (such $H_{0}$ exists by assumption, e.g., $\left.H_{0}=F\right)$. Consider $\Delta H=H_{0}-H_{*}$. Then for $k \in(0,1)$,

$$
\begin{aligned}
W\left(H_{*}+k \Delta H\right) & =W\left((1-k) H_{*}+k H_{0}\right) \\
& =(1-k) W\left(H_{*}\right)+k W\left(H_{0}\right)>k \eta I .
\end{aligned}
$$

Hence, we must have

$$
X^{T}\left[\begin{array}{cc}
0 & (B \Delta H)^{T} \\
B \Delta H & 0
\end{array}\right] X>\eta I .
$$

Since both $B$ and $\Delta H$ are of rank 1, the matrix $\left[\begin{array}{cc}0 & (B \Delta H)^{T} \\ B \Delta H & 0\end{array}\right]$ has at most one positive eigenvalue. For (104) to be true, $X$ can only have one column, i.e., $p=1$.

The optimality of the solution $H_{*}$ means that $\lambda_{\min }(W(H))$ cannot be increased by varying $H$ in a neighborhood of $H_{*}$ along any direction which keeps it within the constraint $H H^{T} \leq 1$, i.e.,

1) $\quad \lambda_{\min }\left(W\left(H_{*}+k \Delta H\right)\right)$ cannot be increased for $k>0$ or $k<0$ if $\Delta H$ is tangential to the sphere surface $H H^{T}=1$ at $H_{*}$;

2) $\quad \lambda_{\min }\left(W\left(H_{*}+k \Delta H\right)\right)$ cannot be increased for $k>0$ if $\Delta H$ points inward of the sphere $H H^{T} \leq 1$ from $H_{*}$.

Partition $X$ as $X=\left[\begin{array}{l}v_{1} \\ v_{2}\end{array}\right]$, where $v_{1}, v_{2} \in \mathbf{R}^{n}$. By the eigenvalue perturbation theory, items 1 ) and 2) reduce to

$$
\begin{aligned}
X^{T} \frac{\partial W\left(H_{*}+k \Delta H\right)}{\partial k} X & =2 v_{2}^{T} B \Delta H v_{1}=0 \\
\text { if } \Delta H H_{*}{ }^{T} & =0 \\
X^{T} \frac{\partial W\left(H_{*}-k H_{*}\right)}{\partial k} X & =-2 v_{2}^{T} B H_{*} v_{1}<0 .
\end{aligned}
$$

For (105), we note that $\lambda_{\min }\left(W\left(H_{*}+k \Delta H\right)\right)$ cannot be increased for either $k>0$ or $k<0$, hence the derivative has to be zero. For (106), we recall that there is one direction $\Delta H=$ $H_{0}-H_{*}$ which makes $\lambda_{\min }\left(W\left(H_{*}+k \Delta H\right)\right)$ strictly increase. Hence, $v_{2}^{T} B H_{*} v_{1} \neq 0$.

Combining (105) and (106), we see that $v_{2}^{T} B \neq 0$ and $v_{1}$ must be proportional to $H_{*}^{T}$. For simplicity, we take $v_{1}=H_{*}^{T}$. Recall that $X=\left[\begin{array}{l}v_{1} \\ v_{2}\end{array}\right]$ is an eigenvector corresponding to the zero eigenvalue of $W\left(H_{*}\right)$, we have

$$
\left[\begin{array}{cc}
I & \left(A+B H_{*}\right)^{T} \\
A+B H_{*} & I
\end{array}\right]\left[\begin{array}{l}
v_{1} \\
v_{2}
\end{array}\right]=0
$$

from which we obtain

$$
\begin{aligned}
v_{2} & =-\left(A+B H_{*}\right) H_{*}{ }^{T} \\
H_{*}{ }^{T} & =\left(A+B H_{*}\right)^{T}\left(A+B H_{*}\right) H_{*}{ }^{T} .
\end{aligned}
$$

Step 3: Construction of $x_{0}$.

From (106) and (107), $v_{1}=H_{*}{ }^{T}$ and $H_{*} H_{*}{ }^{T}=1$, we have

$$
-\left(B^{T} B\right)^{-1} B^{T} A H_{*}^{T}>1 \Longrightarrow F_{0} H_{*}^{T}>1 .
$$

Let $x_{0}=H_{*}{ }^{T}$, then $H_{*} x_{0}=x_{0}^{T} x_{0}=1$ and $\operatorname{sat}\left(F_{0} x_{0}\right)=$ $1=H_{*} x_{0}$. It follows from (108) that

$$
\begin{aligned}
& \left(A x_{0}+B \operatorname{sat}\left(F_{0} x_{0}\right)\right)^{T}\left(A x_{0}+B \operatorname{sat}\left(F_{0} x_{0}\right)\right) \\
& \quad=x_{0}^{T}\left(A+B H_{*}\right)^{T}\left(A+B H_{*}\right) x_{0} \\
& \quad=H_{*}\left(A+B H_{*}\right)^{T}\left(A+B H_{*}\right) H_{*}^{T}=1=x_{0}^{T} P x_{0} .
\end{aligned}
$$

Recall that we have assumed $P=I$ and $\rho_{d}^{*}=1$. This shows that there exists an $x_{0}$ such that $x_{0}^{T} P x_{0}=1$ and $x_{0}$ satisfies (101). It follows that $\rho_{c}^{*} \leq 1=\rho_{d}^{*}$.

From the proof of Lemma 6, we see that at the optimal solution $H_{*}$, the matrix

$$
\left[\begin{array}{cc}
P & \left(A+B H_{*}\right)^{T} \\
A+B H_{*} & P^{-1}
\end{array}\right]
$$

has a single eigenvalue at 0 . This is because $B$ has only one column.

\section{REFERENCES}

[1] M. Arcak, M. Larsen, and P. Kokotovic, "Circle and Popov criteria as tools for nonlinear feedback design," Automatica, vol. 39, pp. 643-650, 2003.

[2] F. Blanchini, "Nonquadratic Lyapunov functions for robust control," $A u$ tomatica, vol. 31, pp. 451-461, 1995.

[3] E. Beran, L. Vandenberghe, and S. Boyd, "A global BMI algorithm based on the generalized benders decomposition," in Proc. European Control Conf., 1997, paper no. 934.

[4] S. Boyd, L. El Ghaoui, E. Feron, and V. Balakrishnan, Linear Matrix Inequalities in Systems and Control Theory. Philadelphia, PA: SIAM, 1994.

[5] R. K. Brayton and C. H. Tong, "Stability of dynamical systems: A constructive approach," IEEE Trans. Circuits Syst., vol. CAS-26, no. 4, pp. 224-234, Apr. 1979.

[6] G. Chesi, A. Garulli, A. Tesi, and A. Vicino, "Homogeneous Lyapunov functions for systems with structured uncertainties," Automatica, vol. 39, pp. 1027-1035, 2003.

[7] Y.-S. Chou, A. L. Tits, and V. Balakrishnan, "Stability multipliers and $\mu$ upper bounds: Connections and implications for numerical verification of frequency domain conditions," IEEE Trans. Autom. Control, vol. 44, no. 5, pp. 906-913, May 1999.

[8] A. Dewey and E. Jury, "A stability inequality for a class of nonlinear feedback systems," IEEE Trans. Autom. Control, vol. AC-11, no. 1, pp. 54-62, Jan. 1966.

[9] W. M. Haddad and V. Kapila, "Absolute stability criteria for multiple slope-restricted monotonic nonlinearities," IEEE Trans. Autom. Control, vol. 40, no. 2, pp. 361-365, Feb. 1995.

[10] R. Goebel, A. R. Teel, T. Hu, and Z. Lin, "Dissipativity for dual linear differential inclusions through conjugate storage functions," in Proc. 43rd IEEE Conf. Decision and Control, 2004, pp. 2700-2705.

[11] K. C. Goh, M. G. Safonov, and G. P. Papavassilopoulos, "A global optimization approach for the BMI problem," in Proc. IEEE Conf. Decision and Control, 1994, pp. 2009-2014.

[12] A. Hassibi, J. How, and S. Boyd, "A path-following method for solving BMI problems in control," in Proc. Amer. Control Conf., 1999, pp. 1385-1389.

[13] T. Hu, B. Huang, and Z. Lin, "Absolute stability with a generalized sector condition," IEEE Trans. Autom. Control, vol. 49, no. 4, pp. 535-548, Apr. 2004.

[14] T. Hu and Z. Lin, Control Systems With Actuator Saturation: Analysis and Design. Boston, MA: Birkhäuser, 2001. 
[15] — - "Exact characterization of invariant ellipsoids for linear systems with saturating actuators," IEEE Trans. Autom. Control, vol. 47, no. 1, pp. 164-169, Jan. 2002.

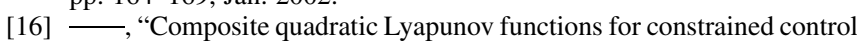
systems," IEEE Trans. Autom. Control, vol. 48, no. 3, pp. 440-450, Mar. 2003.

[17] — , "Properties of composite quadratic Lyapunov functions," IEEE Trans. Autom. Control, vol. 49, no. 7, pp. 1162-1167, Jul. 2004.

[18] T. Hu, Z. Lin, and B. M. Chen, "An analysis and design method for linear systems subject to actuator saturation and disturbance," Automatica, vol. 38 , no. 2, pp. 351-359, 2002.

[19] - "Analysis and design for linear discrete-time systems subject to actuator saturation," Syst. Control Lett., vol. 45, no. 2, pp. 97-112, 2002.

[20] T. Hu, Z. Lin, R. Goebel, and A. R. Teel, "Stability regions for saturated linear systems via conjugate Lyapunov functions," in Proc. 43rd IEEE Conf. Decision and Control, 2004, pp. 5499-5504.

[21] Z. Jarvis-Wloszek and A. K. Packard, "An LMI method to demonstrate simultaneous stability using nonquadratic polynomial Lyapunov functions," in Proc. IEEE Conf. Decision and Control, Las Vegas, NV, 2002, pp. 287-292.

[22] E. I. Jury and B. W. Lee, "On the stability of a certain class of nonlinear sample-data systems," IEEE Trans. Autom. Control, vol. AC-9, no. 1, pp. 51-61, Jan. 1964

[23] M. Johansson and A. Rantzer, "Computation of piecewise quadratic Lyapunov functions for hybrid systems," IEEE Trans. Autom. Control, vol. 43, no. 4, pp. 555-559, Apr. 1998.

[24] A. Molchanov and D. Liu, "Robust absolute stability of time-varying nonlinear discrete-time systems," IEEE Trans. Circuits Syst. I, Fundam. Theory Appl., vol. 49, no. 8, pp. 1129-1137, Aug. 2002.

[25] A. P. Molchanov and Y. Pyatnitskiy, "Criteria of asymptotic stability of differential and difference inclusions encountered in control theory," Syst. Control Lett., vol. 13, pp. 59-64, 1989.

[26] K. S. Narendra and J. Taylor, Frequency Domain Methods for Absolute Stability. New York: Academic, 1973.

[27] J. B. Pearson and J. E. Gibson, "On the asymptotic stability of a class of saturating sampled-data systems," IEEE Trans. Appl. Ind., vol. 7, no. 1, pp. 81-86, Mar. 1964.

[28] H. M. Power and A. C. Tsoi, "Improving the predictions of the circle criterion by combining quadratic forms," IEEE Trans. Autom. Control, vol. AC-28, no. 7, pp. 65-67, Jan. 1973.

[29] V. Singh, "A stability inequality for nonlinear feedback systems with slope-restricted nonlinearity," IEEE Trans. Autom. Control, vol. AC-29, pp. 743-744, 1984.

[30] G. W. Stewart and J. Sun, Matrix Perturbation Theory. Boston, MA: Academic, 1990

[31] T. Wada, M. Ikeda, Y. Ohta, and D. D. Siljak, "Parametric absolute stability of Lur'e systems," IEEE Trans. Autom. Control, vol. 43, no. 11, pp. 1649-1653, Nov. 1998.

[32] J. L. Willems, "The computation of finte stability regions by means of open Lyapunov surfaces," Int. J. Control, vol. 10, no. 5, pp. 537-544, 1969.

[33] L. Xie, S. Shishkin, and M. Fu, "Piecewise Lyapunov functions for robust stability of linear time-varying systems," Syste. Control Lett., vol. 31, pp. 165-171, 1997.
[34] V. A. Yakubovich, "Frequency conditions for the absolute stability of control systems with several nonlinear and linear nonstationary blocks," Autom. Rem. Control, vol. 28, pp. 857-880, 1967.

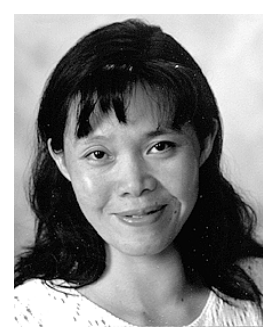

Tingshu Hu received the B.S. and M.S. degrees in electrical engineering from Shanghai Jiao Tong University, Shanghai, China, in 1985 and 1988, respectively, and the $\mathrm{Ph} . \mathrm{D}$. degree in electrical engineering from University of Virginia, Charlottesville, in 2001.

She was a Postdoctoral Researcher at the University of Virginia and the University of California, Santa Barbara. Currently, she is an Assistant Professor with the Department of Electrical and Computer Engineering, the University of Massachusetts, Lowell. Her research interests include nonlinear systems theory, optimization, robust control theory, and control application in mechatronic systems and biomechanical systems. She is a coauthor (with Z. Lin) of the book Control Systems with Actuator Saturation: Analysis and Design (Boston, MA: Birkhäuser, 2001).

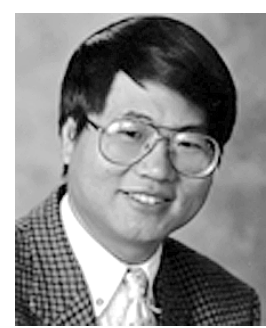

Zongli Lin received the B.S. degree in mathematics and computer science from Xiamen University, Xiamen, China, in 1983, the M.Eng. degree in automatic control from the Chinese Academy of Space Technology, Beijing, in 1989, and the Ph.D. degree in electrical and computer engineering from Washington State University, Pullman, in 1994.

Dr. Lin is currently an Associate Professor with the Charles L. Brown Department of Electrical and Computer Engineering, the University of Virginia, Charlottesville. Previously, he worked as a Control Engineer at the Chinese Academy of Space Technology and as an Assistant Professor with the Department of Applied Mathematics and Statistics, the State University of New York at Stony Brook. His current research interests include nonlinear control, robust control, and modeling and control of magnetic bearing systems In these areas, he has published several papers. He is also the author of the book Low Gain Feedback (London, U.K., Springer-Verlag, 1998), a coauthor (with $\mathrm{T}$. $\mathrm{Hu}$ ) of the book Control Systems with Actuator Saturation: Anabsis and Design (Boston, MA: Birkhäuser, 2001), and a coauthor (with B.M. Chen and Y. Shamash) of the recent book Linear Sjstems Theory: A Structural Decomposition Approach (Boston, MA: Birkhäuser, 2004).

Dr. Lin served as an Associate Editor of the IEEE TRANSACTIONS ON AutOMATIC CONTROL from 2001 to 2003, and is currently an Associate Editor of Automatica and the Corresponding Editor for Conference Activities of the IEEE Control Systems Magazine. He is a Member of the IEEE Control Systems Society's Technical Committee on Nonlinear Systems and Control and heads its Working Group on Control with Constraints. For his work, on control systems with actuator saturation, he received a U.S. Office of Naval Research Young Investigator Award in 1999. 